TITLE:

\title{
Fuzzy Topology and ukasiewicz Logics from the Viewpoint of Duality Theory
}

$\operatorname{AUTHOR}(S)$ :

Maruyama, Yoshihiro

CITATION:

Maruyama, Yoshihiro. Fuzzy Topology and ukasiewicz Logics from the Viewpoint of Duality Theory. Studia Logica 2010, 94(2): 245-269

ISSUE DATE:

2010-03

URL:

http://hdl.handle.net/2433/128930

RIGHT:

The original publication is available at www.springerlink.com; This is not the published version. Please cite only the published version.; この 論文は出版社版でありません。引用の際には出版社版をご確認ご利用 ください。 


\title{
Fuzzy Topology and Łukasiewicz Logics from the Viewpoint of Duality Theory*
}

\author{
Yoshihiro Maruyama \\ Department of Humanistic Informatics \\ Graduate School of Letters \\ Kyoto University, Japan \\ maruyama@i.h.kyoto-u.ac.jp \\ http://researchmap.jp/ymaruyama/
}

\begin{abstract}
This paper explores relationships between many-valued logic and fuzzy topology from the viewpoint of duality theory. We first show a fuzzy topological duality for the algebras of Lukasiewicz $n$-valued logic with truth constants, which generalizes Stone duality for Boolean algebras to the $n$-valued case via fuzzy topology. Then, based on this duality, we show a fuzzy topological duality for the algebras of modal Łukasiewicz $n$-valued logic with truth constants, which generalizes Jónsson-Tarski duality for modal algebras to the $n$-valued case via fuzzy topology. We emphasize that fuzzy topological spaces naturally arise as spectrums of algebras of many-valued logics.
\end{abstract}

Keywords: fuzzy topology; Stone duality; Jónsson-Tarski duality; algebraic logic; many-valued logic; modal logic; Kripke semantics; compactness

\section{Introduction}

This paper aims to explore relationships between many-valued logic and fuzzy topology from the viewpoint of duality theory. In particular, we consider fuzzy topological dualities for the algebras of Lukasiewicz $n$-valued logic $\mathrm{E}_{n}^{c}$ with truth constants and for the algebras of modal Łukasiewicz $n$-valued logic $\mathrm{ME}_{n}^{c}$ with truth constants.

${ }^{*}$ The published version of this paper is in: Studia Logica 94 (2010) 245-269. 
Roughly speaking, a many-valued logic is a logical system in which there are more than two truth values (for a general introduction, see [13, $15,21])$. In many-valued logic, a proposition may have a truth value different from 0 (false) and 1 (true). Łukasiewicz many-valued logic is one of the most prominent many-valued logics. Many-valued logics have often been studied from the algebraic point of view (see, e.g., $[2,6,15]$ ). $\mathrm{MV}$-algebra introduced in [4] provides algebraic semantics for Łukasiewicz infinite-valued logic. $\mathrm{MV}_{n}$-algebra introduced in [14] provides algebraic semantics for Łukasiewicz $n$-valued logic introduced in [20] ([14] also gives an axiomatization of Łukasiewicz $n$-valued logic). $\mathrm{E}_{n}^{c}$-algebra in this paper is considered as $\mathrm{MV}_{n}$-algebra enriched by constants.

Kripke semantics for modal logic is naturally extended to the manyvalued case by allowing for more than two truth values at each possible world and so we can define modal many-valued logics by such many-valued Kripke semantics, including modal Łukasiewicz many-valued logics. Modal many-valued logics have already been studied by several authors (see $[9,10$, $22,29])$.

As a major branch of fuzzy mathematics, fuzzy topology is based on the concept of fuzzy set introduced in [30,11], which is defined by considering many-valued membership function. For example, a $[0,1]$-valued fuzzy set $\mu$ on a set $X$ is defined as a function from $X$ to $[0,1]$. Then, for $x \in X$ and $r \in[0,1], \mu(x)=r$ intuitively means that the proposition " $x \in \mu$ " has a truth value $r$. A fuzzy topology on a set is defined as a collection of fuzzy sets on the set which satisfies some conditions (for details, see Section 3). Historically, Chang [5] introduced the concept of [0, 1]-valued fuzzy topology and thereafter Goguen [12] introduced that of lattice-valued fuzzy topology. There have been many studies on fuzzy topology (see, e.g., [19, 25, 27]).

Stone duality for Boolean algebras (see [17, 28]) is one of the most important results in algebraic logic and states that there is a categorical duality between Boolean algebras (i.e., the algebras of classical propositional logic) and Boolean spaces (i.e., zero-dimensional compact Hausdorff spaces). Since both many-valued logic and fuzzy topology can be considered as based on the idea that there are more than two truth values, it is natural to expect that there is a duality between the algebras of many-valued logic and "fuzzy Boolean spaces." Stone duality for Boolean algebras was extended to Jónsson-Tarski duality (see $[1,3,16,26]$ ) between modal algebras and relational spaces (or descriptive general frames), which is another classical theorem in duality theory. Thus, it is also natural to expect that there is a duality between the algebras of modal many-valued logic and "fuzzy relational spaces." 
In this paper, we realize the above expectations in the cases of $\mathrm{E}_{n}^{c}$ and $\mathrm{ME}_{n}^{c}$. We first develop a categorical duality between the algebras of $\mathrm{E}_{n}^{c}$ and $\mathbf{n}$-fuzzy Boolean spaces (see Definition 4.5), which is a generalization of Stone duality for Boolean algebras to the $\mathbf{n}$-valued case via fuzzy topology. This duality is developed based on the following insights:

- The spectrum of an algebra of $\mathrm{E}_{n}^{c}$ can be naturally equipped with a certain $\mathbf{n}$-fuzzy topology (see Definition 4.9).

- The notion of clopen subset of Boolean space in Stone duality for Boolean algebras corresponds to that of continuous function from $\mathbf{n}$ fuzzy Boolean space to $\mathbf{n}(=\{0,1 /(n-1), 2 /(n-1), \ldots, 1\})$ equipped with the $\mathbf{n}$-fuzzy discrete topology in the duality for the algebras of $\mathrm{E}_{n}^{c}$. This means that the zero-dimensionality of $\mathbf{n}$-fuzzy topological spaces is defined in terms of continuous function into $\mathbf{n}$ (see Definition 4.4).

Moreover, based on the duality for the algebras of $\mathrm{E}_{n}^{c}$, we develop a categorical duality between the algebras of $\mathrm{MŁ}_{n}^{c}$ and $\mathbf{n}$-fuzzy relational spaces (see Definition 6.3), which is a generalization of Jónsson-Tarski duality for modal algebras to the $\mathbf{n}$-valued case via fuzzy topology. Note that an $\mathbf{n}$ fuzzy relational space is also defined in terms of continuous functions into $\mathbf{n}$ (see the items 1 and 2 in the object part of Definition 6.3).

There have been some studies on dualities for algebras of many-valued logics (see, e.g., $[2,7,18,23,24,8,29]$ ). However, they are based on the ordinary topology and therefore do not reveal relationships between manyvalued logic and fuzzy topology. By the results in this paper, we can notice that fuzzy topological spaces naturally arise as spectrums of algebras of some many-valued logics and that there are categorical dualities connecting fuzzy topology and those many-valued logics which generalize Stone and Jónsson-Tarski dualities via fuzzy topology.

This paper is organized as follows. In Section 2, we define $\mathrm{E}_{n}^{c}$ and $\mathrm{E}_{n}^{c}$ algebras, and show basic properties of them. In Section 3, we review basic concepts related to fuzzy topology. In Section 4, we define $\mathbf{n}$-fuzzy Boolean spaces and show a fuzzy topological duality for $\mathrm{E}_{n}^{c}$-algebras, which is a main theorem in this paper. In Section 5, we define $\mathrm{ME}_{n}^{c}$ and $\mathrm{ME}_{n}^{c}$-algebras, and show basic properties of them, including a compactness theorem for $\mathrm{ME}_{n}^{c}$. In Section 6 , we define $\mathbf{n}$-fuzzy relational spaces and show a fuzzy topological duality for $\mathrm{ME}_{n}^{c}$-algebras, which is the other main theorem. 


\section{$2 \quad \mathrm{E}_{n}^{c}$-algebras and basic properties}

Throughout this paper, $n$ denotes a natural number more than 1 .

Definition 2.1. $\mathbf{n}$ denotes $\{0,1 /(n-1), 2 /(n-1), \ldots, 1\}$. We equip $\mathbf{n}$ with all constants $r \in \mathbf{n}$ and the operations $\left(\wedge, \vee, *, \wp, \rightarrow,(-)^{\perp}\right)$ defined as follows:

$$
\begin{aligned}
x \wedge y & =\min (x, y) \\
x \vee y & =\max (x, y) \\
x * y & =\max (0, x+y-1) \\
x \wp y & =\min (1, x+y) \\
x \rightarrow y & =\min (1,1-(x-y)) \\
x^{\perp} & =1-x .
\end{aligned}
$$

We define Eukasiewicz $n$-valued logic with truth constants, which is denoted by $\mathrm{E}_{n}^{c}$. The connectives of $\mathrm{E}_{n}^{c}$ are

$$
\left(\wedge, \vee, *, \wp, \rightarrow,(-)^{\perp}, 0,1 /(n-1), 2 /(n-1), \ldots, 1\right),
$$

where $(\wedge, \vee, *, \wp, \rightarrow)$ are binary connectives, $(-)^{\perp}$ is a unary connective, and $(0,1 /(n-1), 2 /(n-1), \ldots, 1)$ are constants. The formulas of $\mathrm{E}_{n}^{c}$ are recursively defined in the usual way. Let $\mathbf{P V}$ denote the set of propositional variables and Form denote the set of formulas of $\mathrm{E}_{n}^{c}$.

$x \leftrightarrow y$ is the abbreviation of $(x \rightarrow y) \wedge(y \rightarrow x)$. For $m \in \omega$ with $m \neq 0$, $*^{m} x$ is the abbreviation of $x * \ldots * x$ ( $m$-times). For instance, $*^{3} x=x * x * x$.

Definition 2.2. A function $v:$ Form $\rightarrow \mathbf{n}$ is an $\mathbf{n}$-valuation iff it satisfies:

- $v(\varphi @ \psi)=v(\varphi) @ v(\psi)$ for $@=\wedge, \vee, *, \wp, \rightarrow$;

- $v\left(\varphi^{\perp}\right)=(v(\varphi))^{\perp}$;

- $v(r)=r$ for $r \in \mathbf{n}$.

Define $\mathrm{E}_{n}^{c}=\{\varphi \in$ Form ; $v(\varphi)=1$ for any $\mathbf{n}$-valuation $v\}$.

$\mathrm{E}_{n}^{c}$-algebras and homomorphisms are defined as follows.

Definition 2.3. $\left(A, \wedge, \vee, *, \wp, \rightarrow,(-)^{\perp}, 0,1 /(n-1), 2 /(n-1), \ldots, 1\right)$ is an $\mathrm{E}_{n^{-}}^{c}$ algebra iff it satisfies the following set of equations: $\left\{\varphi=\psi ; \varphi \leftrightarrow \psi \in \mathrm{E}_{n}^{c}\right\}$.

A homomorphism of $\mathrm{E}_{n}^{c}$-algebras is defined as a function which preserves the operations $\left(\wedge, \vee, *, \wp, \rightarrow,(-)^{\perp}, 0,1 /(n-1), 2 /(n-1), \ldots, 1\right)$. 
Throughout this paper, we do not distinguish between formulas of $\mathrm{E}_{n}^{c}$ and terms of $\mathrm{E}_{n}^{c}$-algebras.

Definition 2.4. $\varphi \in$ Form is idempotent iff $\varphi * \varphi \leftrightarrow \varphi \in \mathrm{E}_{n}^{c}$.

For an $\mathrm{E}_{n}^{c}$-algebra $A, a \in A$ is idempotent iff $a * a=a$.

$\mathcal{B}(A)$ denotes the set of all idempotent elements of an $\mathrm{七}_{n}^{c}$-algebra $A$.

Let $A$ be an $\mathrm{E}_{n}^{c}$-algebra. Then, we have the following facts: (i) For $a \in A$, $*^{n-1} a$ is always idempotent. (ii) If $a \in A$ is idempotent, then either $v(a)=1$ or $v(a)=0$ holds for any homomorphism $v: A \rightarrow \mathbf{n}$. (iii) If $a, b \in A$ are idempotent, then $a * b=\left(*^{n-1} a\right) *\left(*^{n-1} b\right)=\left(*^{n-1} a\right) \wedge\left(*^{n-1} b\right)=a \wedge b$ and $a \wp b=\left(*^{n-1} a\right) \wp\left(*^{n-1} b\right)=\left(*^{n-1} a\right) \vee\left(*^{n-1} b\right)=a \vee b$.

It is easy to verify the following:

Proposition 2.5. For an $\mathrm{E}_{n}^{c}$-algebra $A, \mathcal{B}(A)$ forms a Boolean algebra. In particular, $a \vee a^{\perp}=1$ for any idempotent element $a$ of $A$.

In the following, we define a formula $\mathrm{T}_{r}(x)$ for $r \in \mathbf{n}$, which intuitively means that the truth value of $x$ is exactly $r$.

Lemma 2.6. Let $A$ be an $\mathrm{E}_{n}^{c}$-algebra and $r \in \mathbf{n}$. There is an idempotent formula $\mathrm{T}_{r}(x)$ with one variable $x$ such that, for any homomorphism $v$ : $A \rightarrow \mathbf{n}$ and any $a \in A$, the following hold:

- $v\left(\mathrm{~T}_{r}(a)\right)=1$ iff $v(a)=r$;

- $v\left(\mathrm{~T}_{r}(a)\right)=0$ iff $v(a) \neq r$.

Proof. If $r=0$, then we can set $\mathrm{T}_{r}(x)=*^{n-1}\left(x^{\perp}\right)$. If $r=1$, then we can set $\mathrm{T}_{r}(x)=*^{n-1} x$.

Let $r=k /(n-1)$ for $k \in\{1, \ldots, n-2\}$. If $k$ is a divisor of $n-1$, then we can set

$$
\mathrm{T}_{r}(x)=*^{n-1}\left(x \leftrightarrow\left(\wp^{\frac{n-1}{k}-1} x\right)^{\perp}\right) .
$$

For a rational number $q$, let $[q]$ denote the greatest integer $n$ such that $n \leq q$. If $k$ is not a divisor of $n-1$, then

$$
\begin{aligned}
v(x)=k /(n-1) \quad & \text { iff } \quad v\left(\wp^{\left[\frac{n-1}{k}\right]} x\right)=\frac{k}{n-1}\left[\frac{n-1}{k}\right](<1) \\
& \text { iff } \quad v\left(\left(\wp^{\left[\frac{n-1}{k}\right]} x\right)^{\perp}\right)=1-\frac{k}{n-1}\left[\frac{n-1}{k}\right] .
\end{aligned}
$$

Since

$$
1-\frac{k}{n-1}\left[\frac{n-1}{k}\right]<\frac{k}{n-1},
$$

this lemma follows by induction on $k$. 
The above lemma is more easily proved by using truth constants $r \in \mathbf{n}$. However, it must be stressed that the above proof works even if we consider Łukasiewicz $n$-valued logic without truth constants.

Note that any homomorphism preserves the operation $\mathrm{T}_{r}(-)$.

Lemma 2.7. Let $A$ be an $\mathrm{E}_{n}^{c}$-algebra and $a_{i} \in A$ for a finite set $I$ and $i \in I$. Then, (i) $\mathrm{T}_{1}\left(\bigvee_{i \in I} a_{i}\right)=\bigvee_{i \in I} \mathrm{~T}_{1}\left(a_{i}\right)$; (ii) $\mathrm{T}_{1}\left(\bigwedge_{i \in I} a_{i}\right)=\bigwedge_{i \in I} \mathrm{~T}_{1}\left(a_{i}\right)$.

Proof. Since $\mathbf{n}$ is totally ordered, we have (i). (ii) is immediate.

By (ii) in the above lemma, $\mathrm{T}_{1}(-)$ is order preserving.

Lemma 2.8. Let $A$ be an $\mathrm{E}_{n}^{c}$-algebra and $r \in \mathbf{n}$. There is an idempotent formula $\mathrm{U}_{r}(x)$ with one variable $x$ such that, for any homomorphism $v$ : $A \rightarrow \mathbf{n}$ and any $a \in A$, the following two conditions hold: (i) $v\left(\mathrm{U}_{r}(a)\right)=1$ iff $v(a) \geq r$; (ii) $v\left(\mathrm{U}_{r}(a)\right)=0$ iff $v(a) \nsupseteq r$.

Proof. It suffices to let $\mathrm{U}_{r}(x)=\bigvee\left\{\mathrm{T}_{s}(x) ; r \leq s\right\}$ by Lemma 2.6.

Note that any homomorphism preserves the operation $\mathrm{U}_{r}(-)$.

Lemma 2.9. Let $A$ be an $\mathrm{E}_{n}^{c}$-algebra and $r \in \mathbf{n}$. There is a formula $S_{r}(x)$ with one variable $x$ such that, for any homomorphism $v: A \rightarrow \mathbf{n}$ and any $a \in A$, the following two conditions hold: (i) $v\left(S_{r}(a)\right)=r$ iff $v(a)=1$; (ii) $v\left(S_{r}(a)\right)=0$ iff $v(a) \neq 1$.

Proof. Let $S_{r}(x)=\left(\mathrm{T}_{1}(x) \rightarrow r\right) \wedge\left(\left(\mathrm{T}_{1}(x)\right)^{\perp} \rightarrow 0\right)$.

Note that any homomorphism preserves the operation $\mathrm{S}_{r}(-)$.

Lemma 2.10. Let $A$ be an $\mathrm{E}_{n}^{c}$-algebra. Let $v$ and $u$ be homomorphisms from $A$ to $\mathbf{n}$. Then, (i) $v=u$ iff (ii) $v^{-1}(\{1\})=u^{-1}(\{1\})$.

Proof. Clearly, (i) implies (ii). We show the converse. Assume that $v^{-1}(\{1\})=$ $u^{-1}(\{1\})$. Suppose for contradiction that $v(a) \neq u(a)$ for some $a \in A$. Let $r=v(a)$. Then $v\left(\mathrm{~T}_{r}(a)\right)=1$ and $u\left(\mathrm{~T}_{r}(a)\right)=0$, which contradicts $v^{-1}(\{1\})=u^{-1}(\{1\})$.

For an $\mathrm{七}_{n}^{c}$-algebra $A$ and $a, b \in A$, we mean $a \vee b=b$ by $a \leq b$.

Lemma 2.11. Let $A$ be an $\mathrm{E}_{n}^{c}$-algebra. For any $a, b \in A$, the following holds:

$$
\bigwedge_{r \in \mathbf{n}}\left(\mathrm{T}_{r}(a) \leftrightarrow \mathrm{T}_{r}(b)\right) \leq a \leftrightarrow b .
$$


Proof. This is proved by straightforward computation.

For a partially ordered set $(M, \leq), X \subset M$ is called an upper set iff if $x \in X$ and $x \leq y$ for $y \in M$ then $y \in X$.

Definition 2.12. Let $A$ be an $\mathrm{E}_{n}^{c}$-algebra. A non-empty subset $F$ of $A$ is called an $\mathbf{n}$-filter of $A$ iff $F$ is an upper set and is closed under $*$. An $\mathbf{n}$-filter $F$ of $A$ is called proper iff $F \neq A$.

An $\mathbf{n}$-filter of $A$ is closed under $\wedge$, since $a * b \leq a \wedge b$ for any $a, b \in A$.

Definition 2.13. Let $A$ be an $\mathrm{E}_{n}^{c}$-algebra. A proper $\mathbf{n}$-filter $P$ of $A$ is prime iff, for any $a, b \in A, a \vee b \in P$ implies either $a \in P$ or $b \in P$.

Proposition 2.14. Let $A$ be an $\mathrm{E}_{n}^{c}$-algebra and $F$ an $\mathbf{n}$-filter of $A$. For $b \in A$, assume $b \notin F$. Then, there is a prime $\mathbf{n}$-filter $P$ of $A$ such that $F \subset P$ and $b \notin P$.

Proof. Let $Z$ be the set of all those n-filters $G$ of $A$ such that $F \subset G$ and $b \notin G$. Then $F \in Z$. Clearly, every chain of $Z$ has an upper bound in $Z$. Thus, by Zorn's lemma, we have a maximal element $P$ in $Z$. Note that $F \subset P$ and $b \notin P$.

To complete the proof, it suffices to show that $P$ is a prime $\mathbf{n}$-filter of $A$. Assume $x \vee y \in P$. Additionally, suppose for contradiction that $x \notin P$ and $y \notin P$. Then, since $P$ is maximal, there exists $\varphi_{x} \in A$ such that $\varphi_{x} \leq b$ and $\varphi_{x}=\left(*^{n-1} x\right) * p_{x}$ for some $p_{x} \in P$. Similary, there exists $\varphi_{y} \in A$ such that $\varphi_{y} \leq b$ and $\varphi_{y}=\left(*^{n-1} y\right) * p_{y}$ for some $p_{y} \in P$. Now, we have the following:

$$
\begin{aligned}
b & \geq\left(\left(*^{n-1} x\right) * p_{x}\right) \vee\left(\left(*^{n-1} y\right) * p_{y}\right) \\
& \geq\left(*^{n-1}\left(x * p_{x}\right)\right) \vee\left(*^{n-1}\left(y * p_{y}\right)\right) \\
& =*^{n-1}\left(\left(x * p_{x}\right) \vee\left(y * p_{y}\right)\right) \\
& \geq *^{n-1}\left(\left(x \vee\left(y * p_{y}\right)\right) *\left(p_{x} \vee\left(y * p_{y}\right)\right)\right) \\
& \geq *^{n-1}\left((x \vee y) * p_{y} * p_{x}\right),
\end{aligned}
$$

where note that $*^{n-1}(x \vee y)=\left(*^{n-1} x\right) \vee\left(*^{n-1} y\right)$ and $x \vee(y * z) \geq(x \vee y) *(x \vee z)$ for any $x, y, z \in A$. Since $p_{x}, p_{y}, x \vee y \in P$, we have $b \in P$, which is a contradiction. Hence $P$ is a prime $\mathbf{n}$-filter of $A$.

We do not use $(-)^{\perp}$ or $\rightarrow$ in the above proof and therefore the above proof works even for algebras of "intuitionistic Łukasiewicz $n$-valued logic."

Definition 2.15. Let $A$ be an $\mathrm{E}_{n}^{c}$-algebra. A subset $X$ of $A$ has finite intersection property (f.i.p.) with respect to $*$ iff, for any $n \in \omega$ with $n \neq 0$, if $a_{1}, \ldots, a_{n} \in X$ then $a_{1} * \ldots * a_{n} \neq 0$. 
Corollary 2.16. Let $A$ be an $\mathrm{E}_{n}^{c}$-algebra and $X$ a subset of $A$. If $X$ has f.i.p. with respect to $*$, then there is a prime $\mathbf{n}$-filter $P$ of $A$ with $X \subset P$.

Proof. By the assumption, we have a proper $\mathbf{n}$-filter $F$ of $A$ generated by $X$. By letting $b=0$ in Proposition 2.14, we have a prime $\mathbf{n}$-filter $P$ of $A$ with $X \subset P$.

Proposition 2.17. Let $A$ be an $\mathrm{E}_{n}^{c}$-algebra. For a prime $\mathbf{n}$-filter $P$ of $A$, define $v_{P}: A \rightarrow \mathbf{n}$ by $v_{P}(a)=r \Leftrightarrow \mathrm{T}_{r}(a) \in P$. Then, $v_{P}$ is a bijection from the set of all prime $\mathbf{n}$-filters of $A$ to the set of all homomorphisms from $A$ to $\mathbf{n}$ with $v_{P}^{-1}(\{1\})=P$.

Proof. Note that $v_{P}$ is well-defined as a function. We prove that $v_{P}$ is a homomorphism. We first show $v_{P}(a * b)=v_{P}(a) * v_{P}(b)$ for $a, b \in A$. Let $r=v_{P}(a)$ and $s=v_{P}(b)$. Then $\mathrm{T}_{r}(a) \in P$ and $\mathrm{T}_{s}(b) \in P$. It is easy to see that $\mathrm{T}_{r}(a) \wedge \mathrm{T}_{s}(b) \leq \mathrm{T}_{r * s}(a * b)$, which intuitively means that if the truth value of $a$ is $r$ and if the truth value of $b$ is $s$ then the truth value of $a * b$ is $r * s$. Since $\mathrm{T}_{r}(a) \in P$ and $\mathrm{T}_{s}(b) \in P$, we have $\mathrm{T}_{r * s}(a * b) \in P$, whence we have $v_{P}(a * b)=r * s=v_{P}(a) * v_{P}(b)$.

Next we show that $v_{P}\left(a^{\perp}\right)=v_{P}(a)^{\perp}$. Let $r=v_{P}(a)$. It is easy to see that $\mathrm{T}_{r}(a) \leq \mathrm{T}_{r^{\perp}}\left(a^{\perp}\right)$. By $\mathrm{T}_{r}(a) \in P$, we have $\mathrm{T}_{r^{\perp}}\left(a^{\perp}\right) \in P$, whence $v_{P}\left(a^{\perp}\right)=r^{\perp}=v_{P}(a)^{\perp}$. As is well-known, $(\wedge, \vee, \wp, \rightarrow)$ can be defined by using only $\left(*,(-)^{\perp}\right)$ (see $\left.[6]\right)$ and so $v_{P}$ preserves the operations $(\wedge, \vee, \wp, \rightarrow)$. Clearly, $v_{P}$ preserves any constant $r \in \mathbf{n}$. Thus, $v_{P}$ is a homomorphism. The remaining part of the proof is straightforward.

\section{3 n-valued fuzzy topology}

Let us review basic concepts from fuzzy set theory and fuzzy topology.

\section{1 n-valued fuzzy set theory}

An $\mathbf{n}$-fuzzy set on a set $S$ is defined as a function from $S$ to $\mathbf{n}$. For $\mathbf{n}$-fuzzy sets $\mu, \lambda$ on $S$, define an $\mathbf{n}$-fuzzy set $\mu @ \lambda$ on $S$ by $(\mu @ \lambda)(x)=\mu(x) @ \lambda(y)$ for $@=\wedge, \vee, *, \wp, \rightarrow$, and define an $\mathbf{n}$-fuzzy set $\mu^{\perp}$ on $S$ by $\left(\mu^{\perp}\right)(x)=(\mu(x))^{\perp}$.

Let $X, Y$ be sets and $f$ a function from $X$ to $Y$. For an $\mathbf{n}$-fuzzy set $\mu$ on $X$, define the direct image $f(\mu): Y \rightarrow \mathbf{n}$ of $\mu$ under $f$ by

$$
f(\mu)(y)=\bigvee\left\{\mu(x) ; x \in f^{-1}(\{y\})\right\} \text { for } y \in Y .
$$


For $f: X \rightarrow Y$ and an $\mathbf{n}$-fuzzy set $\lambda$ on $Y$, define the inverse image $f^{-1}(\lambda): X \rightarrow \mathbf{n}$ of $\lambda$ under $f$ by $f^{-1}(\lambda)=\lambda \circ f$. Note that $f^{-1}$ commutes with $\bigvee$, i.e., $f^{-1}\left(\bigvee_{i \in I} \mu_{i}\right)=\bigvee_{i \in I} f^{-1}\left(\mu_{i}\right)$ for $\mathbf{n}$-fuzzy sets $\mu_{i}$ on $Y$.

For a relation $R$ on a set $S$ and an $\mathbf{n}$-fuzzy set $\mu$ on $S$, define an $\mathbf{n}$ fuzzy set $R^{-1}[\mu]$ on $S$, which is called the inverse image of $\mu$ under $R$, by $R^{-1}[\mu](x)=\bigvee\{\mu(y) ; x R y\}$ for $x \in S$. Note that $R^{-1}\left[\bigvee_{i \in I} \mu_{i}\right]=$ $\bigvee_{i \in I}\left(R^{-1}\left[\mu_{i}\right]\right)$.

\section{$3.2 \quad n$-valued fuzzy topology}

For sets $X$ and $Y, Y^{X}$ denotes the set of all functions from $X$ to $Y$. We do not distinguish between $r \in \mathbf{n}$ and the constant function whose value is always $r$.

Definition 3.1 ([30, 12, 27]). For a set $S$ and a subset $\mathcal{O}$ of $\mathbf{n}^{S},(S, \mathcal{O})$ is an $\mathbf{n}$-fuzzy space iff the following hold:

- $r \in \mathcal{O}$ for any $r \in \mathbf{n}$;

- if $\mu_{1}, \mu_{2} \in \mathcal{O}$ then $\mu_{1} \wedge \mu_{2} \in \mathcal{O}$;

- if $\mu_{i} \in \mathcal{O}$ for $i \in I$ then $\bigvee_{i \in I} \mu_{i} \in \mathcal{O}$,

Then, we call $\mathcal{O}$ the $\mathbf{n}$-fuzzy topology of $(S, \mathcal{O})$, and an element of $\mathcal{O}$ an open $\mathbf{n}$-fuzzy set on $(S, \mathcal{O})$. An $\mathbf{n}$-fuzzy set $\lambda$ on $S$ is a closed $\mathbf{n}$-fuzzy set on $(S, \mathcal{O})$ iff $\lambda=\mu^{\perp}$ for some open $\mathbf{n}$-fuzzy set $\mu$ on $(S, \mathcal{O})$. A clopen $\mathbf{n}$-fuzzy set on $(S, \mathcal{O})$ means a closed and open $\mathbf{n}$-fuzzy set on $(S, \mathcal{O})$.

An $\mathbf{n}$-fuzzy space $(S, \mathcal{O})$ is often denoted by its underlying set $S$.

Definition 3.2. For a set $S, \mathbf{n}^{S}$ is called the discrete $\mathbf{n}$-fuzzy topology on $S .\left(S, \mathbf{n}^{S}\right)$ is called a discrete $\mathbf{n}$-fuzzy space.

Definition 3.3. Let $S_{1}$ and $S_{2}$ be $\mathbf{n}$-fuzzy spaces. Then, $f: S_{1} \rightarrow S_{2}$ is continuous iff, for any open $\mathbf{n}$-fuzzy set $\mu$ on $S_{2}, f^{-1}(\mu)$ (i.e., $\mu \circ f$ ) is an open $\mathbf{n}$-fuzzy set on $S_{1}$.

A composition of continuous functions between $\mathbf{n}$-fuzzy spaces is also continuous (as a function between $\mathbf{n}$-fuzzy spaces).

Definition 3.4. Let $(S, \mathcal{O})$ be an $\mathbf{n}$-fuzzy space. Then, an open basis $\mathcal{B}$ of $(S, \mathcal{O})$ is a subset of $\mathcal{O}$ such that the following holds: (i) $\mathcal{B}$ is closed under $\wedge$; (ii) for any $\mu \in \mathcal{O}$, there are $\mu_{i} \in \mathcal{B}$ for $i \in I$ with $\mu=\bigvee_{i \in I} \mu_{i}$. 
Definition 3.5. An $\mathbf{n}$-fuzzy space $S$ is Kolmogorov iff, for any $x, y \in S$ with $x \neq y$, there is an open $\mathbf{n}$-fuzzy set $\mu$ on $S$ with $\mu(x) \neq \mu(y)$.

Definition 3.6. An $\mathbf{n}$-fuzzy space $S$ is Hausdorff iff, for any $x, y \in S$ with $x \neq y$, there are $r \in \mathbf{n}$ and open $\mathbf{n}$-fuzzy sets $\mu, \lambda$ on $S$ such that $\mu(x) \geq r$, $\lambda(y) \geq r$ and $\mu \wedge \lambda<r$.

Definition $3.7([12])$. Let $S$ be an $\mathbf{n}$-fuzzy space. An $\mathbf{n}$-fuzzy set $\lambda$ on $S$ is compact iff, if $\lambda \leq \bigvee_{i \in I} \mu_{i}$ for open $\mathbf{n}$-fuzzy sets $\mu_{i}$ on $S$, then there is a finite subset $J$ of $I$ such that $\lambda \leq \bigvee_{i \in J} \mu_{i}$.

Let 1 denote the constant function on $S$ whose value is always 1 . Then, $S$ is compact iff, if $1=\bigvee_{i \in I} \mu_{i}$ for open $\mathbf{n}$-fuzzy sets $\mu_{i}$ on $S$, then there is a finite subset $J$ of $I$ such that $1=\bigvee_{i \in J} \mu_{i}$.

We can construct an operation (-)* which turns an $\mathbf{n}$-fuzzy space into a topological space (in the classical sense) as follows.

Definition 3.8. Let $(S, \mathcal{O})$ be an $\mathbf{n}$-fuzzy space. Define

$$
\mathcal{O}^{*}=\left\{\mu^{-1}(\{1\}) ; \mu \in \mathcal{O}\right\} .
$$

Then, $S^{*}$ denotes a topological space $\left(S, \mathcal{O}^{*}\right)$ (see the below proposition).

Lemma 3.9. Let $(S, \mathcal{O})$ be an $\mathbf{n}$-fuzzy space. Then, $S^{*}$ forms a topological space.

Proof. Since $0 \in \mathcal{O}$ and $\emptyset=0^{-1}(\{1\})$, we have $\emptyset \in \mathcal{O}^{*}$. Similarly, $S \in \mathcal{O}^{*}$. Assume $X_{i} \in \mathcal{O}$ for $i \in I$. Then, $X_{i}=\mu_{i}^{-1}(\{1\})$ for some $\mu_{i} \in \mathcal{O}$. Since $\mathbf{n}$ is totally ordered, $\bigcup_{i \in I} X_{i}=\left(\bigvee_{i \in I} \mu_{i}\right)^{-1}(\{1\})$. Thus, by $\bigvee_{i \in I} \mu_{i} \in \mathcal{O}$, we have $\bigcup_{i \in I} X_{i} \in \mathcal{O}^{*}$. It is easy to verify that $X, Y \in \mathcal{O}$ implies $X \cap Y \in \mathcal{O}^{*}$.

\section{A fuzzy topological duality for $\mathrm{E}_{n}^{c}$-algebras}

In this section, we show a fuzzy topological duality for $\mathrm{E}_{n}^{c}$-algebras, which is a generalization of Stone duality for Boolean algebras via fuzzy topology, where note that $\mathrm{E}_{2}^{c}$-algebras coincide with Boolean algebras.

Definition 4.1. $\mathrm{E}_{n}^{c}$-Alg denotes the category whose objects are $\mathrm{E}_{n}^{c}$-algebras and whose arrows are homomorphisms of $\mathrm{E}_{n}^{c}$-algebras.

Our aim in this section is to show that the category $\mathrm{E}_{n}^{c}$-Alg is dually equivalent to the category $\mathrm{FBS}_{n}$, which is defined in the following subsection. 


\subsection{Category $\mathrm{FBS}_{n}$}

We equip $\mathbf{n}$ with the discrete $\mathbf{n}$-fuzzy topology.

Definition 4.2. Let $S$ be an $\mathbf{n}$-fuzzy space. Then, $\operatorname{Cont}(S)$ is defined as the set of all continuous functions from $S$ to $\mathbf{n}$. We endow $\operatorname{Cont}(S)$ with the operations $\left(\wedge, \vee, *, \wp, \rightarrow,(-)^{\perp}, 0,1 /(n-1), 2 /(n-1), \ldots, 1\right)$ defined pointwise: For $f, g \in \operatorname{Cont}(S)$, define $(f @ g)(x)=f(x) @ g(x)$, where @ $=\wedge, \vee, *, \wp, \rightarrow$. For $f \in \operatorname{Cont}(S)$, define $f^{\perp}(x)=(f(x))^{\perp}$. Finally, $r \in \mathbf{n}$ is defined as the constant function on $S$ whose value is always $r$.

We show that the operations of $\operatorname{Cont}(S)$ are well-defined:

Lemma 4.3. Let $S$ be an $\mathbf{n}$-fuzzy space. Then, $\operatorname{Cont}(S)$ is closed under the operations $\left(\wedge, \vee, *, \wp, \rightarrow,(-)^{\perp}, 0,1 /(n-1), \ldots,(n-2) /(n-1), 1\right)$

Proof. For any $r \in \mathbf{n}$, a constant function $r: S \rightarrow \mathbf{n}$ is continuous, since any $s \in \mathbf{n}$ is an open $\mathbf{n}$-fuzzy set on $S$ by Definition 3.1. Then it suffices to show that, if $f, g \in \operatorname{Cont}(S)$, then $f^{\perp}$ and $f @ g$ are continuous for $@=$ $\wedge, \vee, *, \wp, \rightarrow$. Throughout this proof, let $f, g \in \operatorname{Cont}(S)$ and $\mu$ an open $\mathbf{n}$ fuzzy set on $\mathbf{n}$, i.e., a function from $\mathbf{n}$ to $\mathbf{n}$. For $r \in \mathbf{n}$, define $\mu_{r}: \mathbf{n} \rightarrow \mathbf{n}$ by

$$
\mu_{r}(x)= \begin{cases}\mu(r) & \text { if } x=r \\ 0 & \text { otherwise. }\end{cases}
$$

Then, we have $\mu=\bigvee_{r \in \mathbf{n}} \mu_{r}$.

We show that $\left(f^{\perp}\right)^{-1}(\mu)$ is an open $\mathbf{n}$-fuzzy set on $S$. Now, we have

$$
\left(f^{\perp}\right)^{-1}(\mu)=\left(f^{\perp}\right)^{-1}\left(\bigvee_{r \in \mathbf{n}} \mu_{r}\right)=\bigvee_{r \in \mathbf{n}}\left(\left(f^{\perp}\right)^{-1}\left(\mu_{r}\right)\right)
$$

Thus it suffices to show that $\left(f^{\perp}\right)^{-1}\left(\mu_{r}\right)$ is an open $\mathbf{n}$-fuzzy set on $S$ for any $r \in \mathbf{n}$. Define $\lambda_{r}: \mathbf{n} \rightarrow \mathbf{n}$ by

$$
\lambda_{r}(x)= \begin{cases}\mu(r) & \text { if } x=1-r \\ 0 & \text { otherwise }\end{cases}
$$

Then it is straightforward to verify that $\left(f^{\perp}\right)^{-1}\left(\mu_{r}\right)=f^{-1}\left(\lambda_{r}\right)$. Since $f$ is continuous and since $\lambda_{r}$ is an open $\mathbf{n}$-fuzzy set on $\mathbf{n}, f^{-1}\left(\lambda_{r}\right)$ is an open n-fuzzy set on $S$.

Next, we show that $(f * g)^{-1}(\mu)$ is an open $\mathbf{n}$-fuzzy set on $S$. By the same argument as in the case of $f^{\perp}$, it suffices to show that $(f * g)^{-1}\left(\mu_{r}\right)$ is 
an open $\mathbf{n}$-fuzzy set on $S$ for any $r \in \mathbf{n}$. For $p \in \mathbf{n}$, define $\theta_{r, p}: \mathbf{n} \rightarrow \mathbf{n}$ by

$$
\theta_{r, p}(x)= \begin{cases}\mu(r) & \text { if } x=p \\ 0 & \text { otherwise }\end{cases}
$$

For $r \neq 0$, define $\kappa_{r, p}: \mathbf{n} \rightarrow \mathbf{n}$ by

$$
\kappa_{r, p}(x)= \begin{cases}\mu(r) & \text { if } x=r-p+1 \\ 0 & \text { otherwise. }\end{cases}
$$

For $r=0$, define $\kappa_{r, p}: \mathbf{n} \rightarrow \mathbf{n}$ by

$$
\kappa_{r, p}(x)= \begin{cases}\mu(r) & \text { if } x \leq r-p+1 \\ 0 & \text { otherwise. }\end{cases}
$$

Then it is straightforward to verify that

$$
(f * g)^{-1}\left(\mu_{r}\right)=\bigvee_{p \in \mathbf{n}}\left(f^{-1}\left(\theta_{r, p}\right) \wedge g^{-1}\left(\kappa_{r, p}\right)\right)
$$

Since $f, g \in \operatorname{Cont}(S)$, the right-hand side is an open $\mathbf{n}$-fuzzy set on $S$.

As is well-known, $(\wedge, \vee, \wp, \rightarrow)$ can be defined by using only $\left(*,(-)^{\perp}\right)$ (see [6]) and so $(f @ g)^{-1}(\mu)$ is an open $\mathbf{n}$-fuzzy set for $@=\wedge, \vee, \wp, \rightarrow$.

Definition 4.4. For an $\mathbf{n}$-fuzzy space $S, S$ is zero-dimensional iff $\operatorname{Cont}(S)$ forms an open basis of $S$.

Definition 4.5. For an $\mathbf{n}$-fuzzy space $S, S$ is an $\mathbf{n}$-fuzzy Boolean space iff $S$ is zero-dimensional, compact and Kolmogorov.

Definition 4.6. $\mathrm{FBS}_{n}$ is defined as the category of $\mathbf{n}$-fuzzy Boolean spaces and continuous functions.

Proposition 4.7. Let $S$ be an $\mathbf{n}$-fuzzy space. Then, (i) $S$ is an $\mathbf{n}$-fuzzy Boolean space iff (ii) $S$ is zero-dimensional, compact and Hausdorff.

Proof. Cleary, (ii) implies (i). We show the converse. Assume that $S$ is an $\mathbf{n}$-fuzzy Boolean space. It suffices to show that $S$ is Hausdorff. Let $x, y \in S$ with $x \neq y$. Since $S$ is Kolmogorov and since $S$ is zero-dimensional, there is $\mu \in \operatorname{Cont}(S)$ with $\mu(x) \neq \mu(y)$. Let $s=\mu(x)$. Then, $\mathrm{T}_{s} \circ \mu(x)=1$ and $\left(\mathrm{T}_{s} \circ \mu\right)^{\perp}(y)=1$. Since $\mathrm{T}_{s}: \mathbf{n} \rightarrow \mathbf{n}$ is continuous, $\mathrm{T}_{s} \circ \mu \in \operatorname{Cont}(S)$ and $\left(\mathrm{T}_{s} \circ \mu\right)^{\perp} \in \operatorname{Cont}(S)$ by Lemma 4.3. Since $S$ is zero-dimensional, $\mathrm{T}_{s} \circ \mu$ and $\left(\mathrm{T}_{s} \circ \mu\right)^{\perp}$ are open $\mathbf{n}$-fuzzy sets on $S$. We also have $\left(\mathrm{T}_{s} \circ \mu\right) \wedge\left(\mathrm{T}_{s} \circ \mu\right)^{\perp}=0$. Thus, $S$ is Hausdorff. 
Next we show that $(-)^{*}$ turns an $\mathbf{n}$-fuzzy Boolean space into a Boolean space, i.e., a zero-dimensional compact Hausdorff space.

Proposition 4.8. Let $S$ be an $\mathbf{n}$-fuzzy Boolean space. Then, $S^{*}$ forms a Boolean space.

Proof. By Lemma 3.9, $S^{*}$ is a topological space.

First, we show that $S^{*}$ is zero-dimensional in the classical sense. Let $\mathcal{B}^{*}=\left\{\mu^{-1}(\{1\}) ; \mu \in \operatorname{Cont}(S)\right\}$, where, since $S$ is zero-dimensional and so $\mu \in \operatorname{Cont}(S)$ is an open $\mathbf{n}$-fuzzy set on $S, \mu^{-1}(\{1\})$ is an open subset of $S^{*}$. We claim that $\mathcal{B}^{*}$ forms an open basis of $S^{*}$. It is easily verified that $\mathcal{B}^{*}$ is closed under $\cap$. Assume that $O$ is an open subset of $S^{*}$, i.e., $O=\mu^{-1}(\{1\})$ for some open $\mathbf{n}$-fuzzy set $\mu$ on $S$. Since $S$ is zero-dimensional, there are $\mu_{i} \in$ $\operatorname{Cont}(S)$ with $\mu=\bigvee_{i \in I} \mu_{i}$. Since $\mathbf{n}$ is totally ordered, $O=\bigcup_{i \in I} \mu_{i}^{-1}(\{1\})$. It follows from $\mu_{i} \in \operatorname{Cont}(S)$ that $\mu_{i}^{-1}(\{1\}) \in \mathcal{B}^{*}$ for any $i \in I$. This completes the proof of the claim. If $\mu \in \operatorname{Cont}(S)$, then

$$
\left(\mu^{-1}(\{1\})\right)^{c}=\left(\left(\mathrm{T}_{1} \circ \mu\right)^{\perp}\right)^{-1}(\{1\}) .
$$

Since $\mathrm{T}_{1}: \mathbf{n} \rightarrow \mathbf{n}$ is continuous, $\mathrm{T}_{1} \circ \mu \in \operatorname{Cont}(S)$, whence, by Lemma 4.3, $\left(\mathrm{T}_{1} \circ \mu\right)^{\perp} \in \operatorname{Cont}(S)$. Thus the right-hand side is open in $S^{*}$ and so $\mu^{-1}(\{1\})$ is clopen in $S^{*}$ for $\mu \in \operatorname{Cont}(S)$. Hence, $S^{*}$ is zero-dimensional.

Second, we show that $S^{*}$ is compact in the classical sense. Assume that $S^{*}=\bigcup_{i \in I} O_{i}$ for some open subsets $O_{i}$ of $S^{*}$. Since $\mathcal{B}^{*}$ forms an open basis of $S^{*}$, we may assume that $S^{*}=\bigcup_{i \in I} \mu_{i}^{-1}(\{1\})$ for some $\mu_{i} \in \operatorname{Cont}(S)$. Then, $1=\bigvee_{i \in I} \mu_{i}$ where 1 denotes the constant function on $S\left(=S^{*}\right)$ whose value is always 1 . Since $S$ is zero-dimensional, $\mu_{i}$ is an open $\mathbf{n}$-fuzzy set on $S$. Thus, since $S$ is compact, there is a finite subset $J$ of $I$ such that $1=\bigvee_{j \in J} \mu_{j}$, whence $S^{*}=\bigcup_{j \in J} \mu_{j}^{-1}(\{1\})$. Hence $S^{*}$ is compact.

Finally, we show that $S^{*}$ is Hausdorff in the classical sense. Since $S^{*}$ is zero-dimensional, it suffices to show that $S^{*}$ is Kolmogorov in the classical sense. Assume $x, y \in S^{*}$ with $x \neq y$. Since $S$ is Kolmogorov, there is an open $\mathbf{n}$-fuzzy set $\mu$ on $S$ with $\mu(x) \neq \mu(y)$. Since $S$ is zero-dimensional, $\mu=\bigvee_{i \in I} \mu_{i}$ for some $\mu_{i} \in \operatorname{Cont}(S)$. There is $i \in I$ with $\mu_{i}(x) \neq \mu_{i}(y)$. Let $r=\mu_{i}(x)$. Then, we have $\mathrm{T}_{r} \circ \mu_{i}(x)=1$ and $\mathrm{T}_{r} \circ \mu_{i}(y)=0$, whence we have $x \in\left(\mathrm{T}_{r} \circ \mu_{i}\right)^{-1}(\{1\})$ and $y \notin\left(\mathrm{T}_{r} \circ \mu_{i}\right)^{-1}(\{1\})$. Since $\mathrm{T}_{r}: \mathbf{n} \rightarrow \mathbf{n}$ is continuous, it follows from $\mu_{i} \in \operatorname{Cont}(S)$ that $\mathrm{T}_{r} \circ \mu_{i} \in \operatorname{Cont}(S)$, whence $\mathrm{T}_{r} \circ \mu_{i}$ is an open $\mathbf{n}$-fuzzy set on $S$ and so $\left(\mathrm{T}_{r} \circ \mu_{i}\right)^{-1}(\{1\})$ is an open subset of $S^{*}$. Hence $S^{*}$ is Kolmogorov.

\subsection{Functors Spec and Cont}

We define the spectrum $\operatorname{Spec}(A)$ of an $\mathrm{E}_{n}^{c}$-algebra $A$ as follows. 
Definition 4.9. For an $\mathrm{E}_{n}^{c}$-algebra $A, \operatorname{Spec}(A)$ is defined as the set of all homomorphisms (of $\mathrm{E}_{n}^{c}$-algebras) from $A$ to $\mathbf{n}$ equipped with the $\mathbf{n}$-fuzzy topology generated by $\{\langle a\rangle ; a \in A\}$, where $\langle a\rangle: \operatorname{Spec}(A) \rightarrow \mathbf{n}$ is defined by

$$
\langle a\rangle(v)=v(a) .
$$

The operations $\left(\wedge, \vee, *, \wp, \rightarrow,(-)^{\perp}\right)$ on $\{\langle a\rangle ; a \in A\}$ are defined pointwise as in Definition 4.2 .

$\{\langle a\rangle ; a \in A\}$ forms an open basis of $\operatorname{Spec}(A)$, since $\langle a\rangle \wedge\langle b\rangle=\langle a \wedge b\rangle$.

Definition 4.10. We define a contravariant functor Spec $: \mathrm{七}_{n}^{c}$-Alg $\rightarrow \mathrm{FBS}_{n}$.

For an object $A$ in $\mathrm{E}_{n}^{c}$-Alg, define $\operatorname{Spec}(A)$ as in Definition 4.9.

For an arrow $f: A_{1} \rightarrow A_{2}$ in $\mathrm{E}_{n}^{c}$-Alg, define $\operatorname{Spec}(f): \operatorname{Spec}\left(A_{2}\right) \rightarrow$ $\operatorname{Spec}\left(A_{1}\right)$ by $\operatorname{Spec}(f)(v)=v \circ f$ for $v \in \operatorname{Spec}\left(A_{2}\right)$.

The well-definedness of the functor Spec is proved by Proposition 4.15 and Proposition 4.16 below.

Since $\mathbf{n}$ is a totally ordered complete lattice, we have:

Lemma 4.11. Let $\mu_{i}$ be an $\mathbf{n}$-fuzzy set on a set $S$ for a set $I$ and $i \in I$. Then, (i) $\mathrm{T}_{1} \circ \bigvee_{i \in I} \mu_{i}=\bigvee_{i \in I}\left(\mathrm{~T}_{1} \circ \mu_{i}\right)$; (ii) $\mathrm{T}_{1} \circ \bigwedge_{i \in I} \mu_{i}=\bigwedge_{i \in I}\left(\mathrm{~T}_{1} \circ \mu_{i}\right)$.

Lemma 4.12. Let $A$ be an $\mathrm{E}_{n}^{c}$-algebra. Then, $\operatorname{Spec}(A)$ is compact.

Proof. Assume that $1=\bigvee_{j \in J} \mu_{j}$ for open $\mathbf{n}$-fuzzy sets $\mu_{j}$ on $\operatorname{Spec}(A)$, where 1 denotes the constant function defined on $\operatorname{Spec}(A)$ whose value is always 1. Then, since $\{\langle a\rangle ; a \in A\}$ is an open basis of $\operatorname{Spec}(A)$, we may assume that $1=\bigvee_{i \in I}\left\langle a_{i}\right\rangle$ for some $a_{i} \in A$. It follows from Lemma 4.11 that $1=\mathrm{T}_{1} \circ 1=\mathrm{T}_{1} \circ \bigvee_{i \in I}\left\langle a_{i}\right\rangle=\bigvee_{i \in I} \mathrm{~T}_{1} \circ\left\langle a_{i}\right\rangle=\bigvee_{i \in I}\left\langle\mathrm{~T}_{1}\left(a_{i}\right)\right\rangle$. Thus, we have

$$
0=\left(\bigvee_{i \in I}\left\langle\mathrm{~T}_{1}\left(a_{i}\right)\right\rangle\right)^{\perp}=\bigwedge_{i \in I}\left\langle\left(\mathrm{~T}_{1}\left(a_{i}\right)\right)^{\perp}\right\rangle
$$

Then, there is no homomorphism $v: A \rightarrow \mathbf{n}$ such that $v\left(\left(\mathrm{~T}_{1}\left(a_{i}\right)\right)^{\perp}\right)=$ 1 for any $i \in I$. Therefore, by Proposition 2.17, there is no prime $\mathbf{n}$ filter of $A$ which contains $\left\{\left(\mathrm{T}_{1}\left(a_{i}\right)\right)^{\perp} ; i \in I\right\}$. Thus, by Corollary 2.16, $\left\{\left(\mathrm{T}_{1}\left(a_{i}\right)\right)^{\perp} ; i \in I\right\}$ does not have f.i.p. with respect to $*$ and so there is a finite subset $\left\{i_{1}, \ldots i_{m}\right\}$ of $I$ such that $\left(\mathrm{T}_{1}\left(a_{i_{1}}\right)\right)^{\perp} * \ldots *\left(\mathrm{~T}_{1}\left(a_{i_{m}}\right)\right)^{\perp}=0$, whence $\mathrm{T}_{1}\left(a_{i_{1}}\right) \wp \ldots \wp \mathrm{T}_{1}\left(a_{i_{m}}\right)=1$. Since $\mathrm{T}_{1}\left(a_{i_{k}}\right)$ is idempotent for any $k \in\{1, \ldots, m\}$, we have $\mathrm{T}_{1}\left(a_{i_{1}}\right) \vee \ldots \vee \mathrm{T}_{1}\left(a_{i_{m}}\right)=1$ and, by Lemma $2.7, \mathrm{~T}_{1}\left(a_{i_{1}} \vee \ldots \vee a_{i_{m}}\right)=1$. By $\mathrm{T}_{1}(x) \leq x$, we have $a_{i_{1}} \vee \ldots \vee a_{i_{m}}=1$, whence $\left\langle a_{i_{1}} \vee \ldots \vee a_{i_{m}}\right\rangle=1$. This completes the proof. 
Lemma 4.13. Let $A$ be an $\mathrm{E}_{n}^{c}$-algebra. Then, $\operatorname{Spec}(A)$ is Kolmogorov.

Proof. Let $v_{1}, v_{2} \in \operatorname{Spec}(A)$ with $v_{1} \neq v_{2}$. Then there is $a \in A$ such that $v_{1}(a) \neq v_{2}(a)$, whence we have $\langle a\rangle\left(v_{1}\right) \neq\langle a\rangle\left(v_{2}\right)$.

Lemma 4.14. Let $A$ be an $\mathrm{E}_{n}^{c}$-algebra. Then, $\operatorname{Spec}(A)$ is zero-dimensional.

Proof. Since $\{\langle a\rangle ; a \in A\}$ forms an open basis of $\operatorname{Spec}(A)$, it suffices to show that

$$
\text { Cont } \circ \operatorname{Spec}(A)=\{\langle a\rangle ; a \in A\} .
$$

We first show that $\operatorname{Cont} \circ \operatorname{Spec}(A) \supset\{\langle a\rangle ; a \in A\}$, i.e., $\langle a\rangle$ is continuous for any $a \in A$. Let $a \in A$ and $\mu$ an $\mathbf{n}$-fuzzy set on $\mathbf{n}$. Then, by Lemma 2.9,

$$
\langle a\rangle^{-1}(\mu)=\mu \circ\langle a\rangle=\bigvee_{r \in \mathbf{n}}\left(\mathrm{S}_{\mu(r)} \circ \mathrm{T}_{r}\right) \circ\langle a\rangle=\left\langle\bigvee_{r \in \mathbf{n}}\left(\mathrm{S}_{\mu(r)}\left(\mathrm{T}_{r}(a)\right)\right)\right\rangle .
$$

Hence $\langle a\rangle$ is continuous.

Next we show Cont $\circ \operatorname{Spec}(A) \subset\{\langle a\rangle ; a \in A\}$. Let $f \in \operatorname{Cont} \circ \operatorname{Spec}(A)$ and $r \in \mathbf{n}$. Define an $\mathbf{n}$-fuzzy set $\lambda_{r}$ on $\mathbf{n}$ by $\lambda_{r}(x)=1$ for $x=r$ and $\lambda_{r}(x)=0$ for $x \neq r$. Since $f$ is continuous, $f^{-1}\left(\lambda_{r}\right)=\bigvee_{i \in I}\left\langle a_{i}\right\rangle$ for some $a_{i} \in A$. Now the following holds:

$$
1=f^{-1}\left(\lambda_{r}\right) \vee\left(f^{-1}\left(\lambda_{r}\right)\right)^{\perp}=\left(\bigvee_{i \in I}\left\langle a_{i}\right\rangle\right) \vee\left(f^{-1}\left(\lambda_{r}\right)\right)^{\perp} .
$$

Here, we have $\left(f^{-1}\left(\lambda_{r}\right)\right)^{\perp}=\left(\lambda_{r} \circ f\right)^{\perp}=\lambda_{r}{ }^{\perp} \circ f=f^{-1}\left(\lambda_{r}{ }^{\perp}\right)$. Since $f^{-1}\left(\lambda_{r}{ }^{\perp}\right)$ is an open $\mathbf{n}$-fuzzy set, $\left(f^{-1}\left(\lambda_{r}\right)\right)^{\perp}$ is an open $\mathbf{n}$-fuzzy set on $\operatorname{Spec}(A)$. Since $\operatorname{Spec}(A)$ is compact by Lemma 4.12, there is a finite subset $J$ of $I$ such that $1=\left(\bigvee_{j \in J}\left\langle a_{j}\right\rangle\right) \vee\left(f^{-1}\left(\lambda_{r}\right)\right)^{\perp}$. Thus, $f^{-1}\left(\lambda_{r}\right) \leq \bigvee_{j \in J}\left\langle a_{j}\right\rangle$. Since $\bigvee_{j \in J}\left\langle a_{j}\right\rangle \leq \bigvee_{i \in I}\left\langle a_{i}\right\rangle=f^{-1}\left(\lambda_{r}\right)$, we have $f^{-1}\left(\lambda_{r}\right)=\bigvee_{j \in J}\left\langle a_{j}\right\rangle$. Since $J$ is finite, $f^{-1}\left(\lambda_{r}\right)=\bigvee_{j \in J}\left\langle a_{j}\right\rangle=\left\langle\bigvee_{j \in J} a_{j}\right\rangle$. Let $a_{r}=\bigvee_{j \in J} a_{j}$. Note that if $v \in f^{-1}(\{r\})$ then $v\left(a_{r}\right)=1$ and that if $v \notin f^{-1}(\{r\})$ then $v\left(a_{r}\right)=0$. We claim that $f=\left\langle\bigvee_{r \in \mathbf{n}}\left(r \wedge a_{r}\right)\right\rangle$. If $v \in f^{-1}(\{s\})$ for $s \in \mathbf{n}$, then

$$
\left\langle\bigvee_{r \in \mathbf{n}}\left(r \wedge a_{r}\right)\right\rangle(v)=v\left(\bigvee_{r \in \mathbf{n}}\left(r \wedge a_{r}\right)\right)=\bigvee_{r \in \mathbf{n}}\left(r \wedge v\left(a_{r}\right)\right)=s=f(v)
$$

This completes the proof.

By the above lemmas, we obtain the following proposition.

Proposition 4.15. Let $A$ be an object in $\mathrm{E}_{n}^{c}$-Alg. Then, $\operatorname{Spec}(A)$ is an object in the category $\mathrm{FBS}_{n}$. 
Proposition 4.16. Let $A_{1}$ and $A_{2}$ be objects in $\mathrm{E}_{n}^{c}$-Alg and $f: A_{1} \rightarrow A_{2}$ an arrow in $\mathrm{E}_{n}^{c}$-Alg. Then, $\operatorname{Spec}(f)$ is an arrow in $\mathrm{FBS}_{n}$.

Proof. Since the inverse image $(\operatorname{Spec}(f))^{-1}$ commutes with $\bigvee$, it suffices to show that $(\operatorname{Spec}(f))^{-1}(\langle a\rangle)$ is an open $\mathbf{n}$-fuzzy set on $\operatorname{Spec}\left(A_{2}\right)$ for any $a \in A_{1}$. For $v \in \operatorname{Spec}\left(A_{2}\right)$, we have

$\left(\operatorname{Spec}(f)^{-1}(\langle a\rangle)\right)(v)=\langle a\rangle \circ \operatorname{Spec}(f)(v)=\langle a\rangle(v \circ f)=v \circ f(a)=\langle f(a)\rangle(v)$.

Hence $(\operatorname{Spec}(f))^{-1}(\langle a\rangle)=\langle f(a)\rangle$, which is an open $\mathbf{n}$-fuzzy set.

Definition 4.17. We define a contravariant functor Cont $: \mathrm{FBS}_{n} \rightarrow \mathrm{E}_{n}^{c}$-Alg.

For an object $S$ in $\mathrm{FBS}_{n}$, Cont $(S)$ is defined as in Definition 4.2.

For an arrow $f: S \rightarrow T$ in $\operatorname{FBS}_{n}, \operatorname{Cont}(f): \operatorname{Cont}(T) \rightarrow \operatorname{Cont}(S)$ is defined by $\operatorname{Cont}(f)(g)=g \circ f$ for $g \in \operatorname{Cont}(T)$.

Since the operations of $\operatorname{Cont}(S)$ are defined pointwise, $\operatorname{Cont}(S)$ is an $\mathrm{E}_{n}^{c}$-algebra and the following holds, whence Cont is well-defined.

Proposition 4.18. Let $S_{1}$ and $S_{2}$ be objects in $\mathrm{FBS}_{n}$, and $f: S_{1} \rightarrow S_{2}$ an arrow in $\mathrm{FBS}_{n}$. Then, Cont $(f)$ is an arrow in $\mathrm{E}_{n}^{c}$-Alg.

Definition 4.19. Let $A$ be an $\mathrm{E}_{n}^{c}$-algebra. Then, $\operatorname{Spec}_{2}(\mathcal{B}(A))$ is defined as the set of all homomorphisms of Boolean algebras from $\mathcal{B}(A)$ to 2 equipped with the (ordinary) topology generated by $\left\{\langle a\rangle_{\mathbf{2}} ; a \in \mathcal{B}(A)\right\}$, where $\langle a\rangle_{\mathbf{2}}=$ $\left\{v \in \operatorname{Spec}_{\mathbf{2}}(\mathcal{B}(A)) ; v(a)=1\right\}$.

Proposition 4.20. Let $A$ be an $\mathrm{E}_{n}^{c}$-algebra. Define a function $t_{1}$ from $\operatorname{Spec}(A)^{*}$ to $\operatorname{Spec}_{2}(\mathcal{B}(A))$ by $t_{1}(v)=\mathrm{T}_{1} \circ v$. Then, $t_{1}$ is a homeomorphism.

Proof. By Lemma 2.10, $t_{1}$ is injective. We show that $t_{1}$ is surjective. Let $v \in \operatorname{Spec}_{2}(\mathcal{B}(A))$. Define $u \in \operatorname{Spec}(A)$ by $u(a)=r \Leftrightarrow \mathrm{T}_{r}(a) \in v^{-1}(\{1\})$ for $a \in A$, where note $\mathrm{T}_{r}(a) \in \mathcal{B}(A)$. Then, in a similar way to Proposition 2.17 , it is verified that $u$ is a homomorphism (i.e., $u \in \operatorname{Spec}(A)$ ). Moreover, we have $t_{1}(u)=v$ on $\mathcal{B}(A)$. Thus $t_{1}$ is bijective. It is straightforward to verify the remaining part of the proof. Note that, for $\langle a\rangle_{\mathbf{n}}=\{v \in$ $\operatorname{Spec}(A) ; v(a)=1\},\left\{\langle a\rangle_{\mathbf{n}} ; a \in A\right\}$ forms an open basis of $\operatorname{Spec}(A)^{*}$ and that $t_{1}\left(\langle a\rangle_{\mathbf{n}}\right)=\left\langle\mathrm{T}_{1}(a)\right\rangle_{\mathbf{2}}$ for $a \in A$.

\subsection{A fuzzy topological duality for $\mathrm{E}_{n}^{c}$-algebras}

Theorem 4.21. Let $A$ be an $\mathrm{E}_{n}^{c}$-algebra. Then, there is an isomorphism between $A$ and Cont $\circ \operatorname{Spec}(A)$ in the category $\mathrm{L}_{n}^{c}$-Alg. 
Proof. Define $\langle-\rangle: A \rightarrow$ Cont $\circ \operatorname{Spec}(A)$ as in Definition 4.9. In the proof of Lemma 4.14, it has already been proven that $\langle-\rangle$ is well-defined and surjective. Since the operations of $\operatorname{Cont} \circ \operatorname{Spec}(A)$ are defined pointwise, $\langle-\rangle$ is a homomorphism.

Thus it suffices to show that $\langle-\rangle$ is injective. Assume that $\langle a\rangle=\langle b\rangle$ for $a, b \in A$, which means that, for any $v \in \operatorname{Spec}(A)$, we have $v(a)=v(b)$. Thus, for any $v \in \operatorname{Spec}(A)$ and any $r \in \mathbf{n}$, we have $v\left(\mathrm{~T}_{r}(a)\right)=v\left(\mathrm{~T}_{r}(b)\right)$. Thus, it follows from Proposition 2.17 that, for any prime $\mathbf{n}$-filter $P$ of $A$ and any $r \in \mathbf{n}, \mathrm{T}_{r}(a) \in P$ iff $\mathrm{T}_{r}(b) \in P$.

We claim that $\mathrm{T}_{r}(a)=\mathrm{T}_{r}(b)$ for any $r \in \mathbf{n}$. Suppose for contradiction that $\mathrm{T}_{r}(a) \neq \mathrm{T}_{r}(b)$ for some $r \in \mathbf{n}$. We may assume without loss of generality that $\mathrm{T}_{r}(a) \not \leq \mathrm{T}_{r}(b)$. Let $F=\left\{x \in A ; \mathrm{T}_{r}(a) \leq x\right\}$. Then, since $\mathrm{T}_{r}(a)$ is idempotent, $F$ is an $\mathbf{n}$-filter of $A$. Cleary, $\mathrm{T}_{r}(b) \notin F$. Thus, by Lemma 2.14 , there is a prime $\mathbf{n}$-filter $P$ of $A$ such that $F \subset P$ and $\mathrm{T}_{r}(b) \notin P$. By $F \subset P$, we have $\mathrm{T}_{r}(a) \in P$, which contradicts $\mathrm{T}_{r}(b) \notin P$, since we have already shown that $\mathrm{T}_{r}(a) \in P$ iff $\mathrm{T}_{r}(b) \in P$. Thus, $\mathrm{T}_{r}(a)=\mathrm{T}_{r}(b)$ for any $r \in \mathbf{n}$, whence $\bigwedge_{r \in \mathbf{n}}\left(\mathrm{T}_{r}(a) \leftrightarrow \mathrm{T}_{r}(b)\right)=1$. Hence, it follows from Lemma 2.11 that $a=b$, and therefore $\langle-\rangle$ is injective.

Theorem 4.22. Let $S$ be an $\mathbf{n}$-fuzzy Boolean space. Then, there is an isomorphism between $S$ and $\operatorname{Spec} \circ \operatorname{Cont}(S)$ in the category $\mathrm{FBS}_{n}$.

Proof. Define $\Psi: S \rightarrow$ Spec $\circ \operatorname{Cont}(S)$ by $\Psi(x)(f)=f(x)$ for $x \in S$ and $f \in \operatorname{Cont}(S)$. Since the operations of $\operatorname{Cont}(S)$ are defined pointwise, $\Psi(x)$ is a homomorphism and so $\Psi$ is well-defined.

We show that $\Psi$ is continuous. Let $f \in \operatorname{Cont}(S)$. Then $\Psi^{-1}(\langle f\rangle)=f$ by the following:

$$
\left(\Psi^{-1}(\langle f\rangle)\right)(x)=\langle f\rangle \circ \Psi(x)=\Psi(x)(f)=f(x) .
$$

Since $f \in \operatorname{Cont}(S)$ and $S$ is zero-dimensional, $f$ is an an open $\mathbf{n}$-fuzzy set and so $\Psi^{-1}(\langle f\rangle)$ is an open $\mathbf{n}$-fuzzy set on $S$. Since the inverse image $\Psi^{-1}$ commutes with $\bigvee$, it follows that $\Psi$ is continuous.

Next we show that $\Psi$ is injective. Let $x, y \in S$ with $x \neq y$. Since $S$ is Kolmogorov and zero-dimensional, there is $f \in \operatorname{Cont}(S)$ with $f(x) \neq f(y)$. Thus, $\Psi(x)(f)=f(x) \neq f(y)=\Psi(y)(f)$, whence $\Psi$ is injective.

Next we show that $\Psi$ is surjective. Let $v \in \operatorname{Spec} \circ \operatorname{Cont}(S)$. Consider $\left\{f^{-1}(\{1\}) ; v(f)=1\right\}$. Define $\mu: \mathbf{n} \rightarrow \mathbf{n}$ by $\mu(1)=0$ and $\mu(x)=1$ for $x \neq 1$. Since $f^{-1}(\mu)(=\mu \circ f)$ is an open $\mathbf{n}$-fuzzy set on $S$ for $f \in \operatorname{Cont}(S)$, $(\mu \circ f)^{-1}(\{1\})$ is an open subset of $S^{*}$. Since $(\mu \circ f)^{-1}(\{1\})=\left(f^{-1}(\{1\})\right)^{c}$, $f^{-1}(\{1\})$ is a closed subset of $S^{*}$ for $f \in \operatorname{Cont}(S)$. 
We claim that $\left\{f^{-1}(\{1\}) ; v(f)=1\right\}$ has the finite intersection property. Since $f^{-1}(\{1\}) \cap g^{-1}(\{1\})=(f \wedge g)^{-1}(\{1\})$ for $f, g \in \operatorname{Cont}(S)$, it suffices to show that if $v(f)=1$ then $f^{-1}(\{1\})$ is not empty. Suppose for contradiction that $v(f)=1$ and $f^{-1}(\{1\})=\emptyset$. Since $f^{-1}(\{1\})=\emptyset$, we have $\mathrm{T}_{1}(f)=0$. Thus $v\left(\mathrm{~T}_{1}(f)\right)=0$ and so $v(f) \neq 1$, which contradicts $v(f)=1$.

By Proposition $4.8, S^{*}$ is compact. Thus, there is $z \in S$ such that $z \in \bigcap\left\{f^{-1}(\{1\}) ; v(f)=1\right\}$. We claim that $\Psi(z)=v$. By the definition of $z$, if $v(f)=1$ then $\Psi(z)(f)=1$. We show the converse. Suppose for constradiction that $\Psi(z)(f)=1$ and $v(f) \neq 1$. Then $v\left(\mathrm{~T}_{1}(f)\right)=\mathrm{T}_{1}(v(f))=$ 0 and so $v\left(\left(\mathrm{~T}_{1}(f)\right)^{\perp}\right)=1$. By the definition of $z,\left(\mathrm{~T}_{1}(f)\right)^{\perp}(z)=1$ and so $\left(\mathrm{T}_{1}(f)\right)(z)=0$. Thus $f(z) \neq 1$, which contradicts $\Psi(z)(f)=1$. Hence, for any $f \in \operatorname{Cont}(S), v(f)=1$ iff $\Psi(z)(f)=1$. By Lemma 2.10, we have $\Psi(z)=v$. Hence, $\Psi$ is surjective.

Finally we show that $\Psi^{-1}$ is an arrow in the category $\mathrm{FBS}_{n}$. It suffices to show that, for any open $\mathbf{n}$-fuzzy set $\lambda$ on $S, \Psi(\lambda)$ is an open $\mathbf{n}$-fuzzy set on $\operatorname{Spec} \circ \operatorname{Cont}(S)$. Since $S$ is zero-dimensional, there are $f_{i} \in \operatorname{Cont}(S)$ with $\lambda=\bigvee_{i \in I} f_{i}$. For $v \in \operatorname{Spec} \circ \operatorname{Cont}(S)$, the following holds:

$\Psi(\lambda)(v)=\bigvee\left\{\lambda(x) ; x \in \Psi^{-1}(\{v\})\right\}=\lambda(z)=v(\lambda)=v\left(\bigvee_{i \in I} f_{i}\right)=\left(\bigvee_{i \in I}\left\langle f_{i}\right\rangle\right)(v)$,

where $z$ is defined as the unique element $x$ such that $\Psi(x)=v$ (for the definition of the direct image of an $\mathbf{n}$-fuzzy set, see Subsection 3.1). Hence $\Psi(\lambda)=\bigvee_{i \in I}\left\langle f_{i}\right\rangle$ and so $\Psi(\lambda)$ is an open $\mathbf{n}$-fuzzy set on $\operatorname{Spec} \circ \operatorname{Cont}(S)$.

By Theorem 4.21 and Theorem 4.22, we obtain a fuzzy topological duality for $\mathrm{E}_{n}^{c}$-algebras, which is a generalization of Stone duality for Boolean algebras to the $n$-valued case via fuzzy topology.

Theorem 4.23. The category $\mathrm{E}_{n}^{c}$-Alg is dually equivalent to the category $\mathrm{FBS}_{n}$ via the functors Spec and Cont.

Proof. Let $\mathrm{Id}_{1}$ denote the identity functor on $\mathrm{E}_{n}^{c}$-Alg and $\mathrm{Id}_{2}$ denote the identity functor on $\mathrm{FBS}_{n}$. Then, we define two natural transformations $\epsilon: \mathrm{Id}_{1} \rightarrow$ Cont $\circ$ Spec and $\eta: \mathrm{Id}_{2} \rightarrow$ Spec $\circ$ Cont. For an $\mathrm{E}_{n}^{c}$-algebra $A$, define $\epsilon_{A}: A \rightarrow \operatorname{Cont} \circ \operatorname{Spec}(A)$ by $\epsilon_{A}=\langle-\rangle$ (see Theorem 4.21). For an $\mathbf{n}$-fuzzy Boolean space $S$, define $\eta_{S}: S \rightarrow \operatorname{Spec} \circ \operatorname{Cont}(S)$ by $\eta_{S}=\Psi$ (see Theorem 4.22). It is straightforward to see that $\eta$ and $\epsilon$ are natural transformations. By Theorem 4.21 and Theorem 4.22, $\eta$ and $\epsilon$ are natural isomorphisms. 


\section{$5 \quad \mathrm{MŁ}_{n}^{c}$-algebras and basic properties}

We define modal Łukasiewicz $n$-valued logic with truth constants $\mathrm{ME}_{n}^{c}$ by $\mathbf{n}$-valued Kripke semantics. The connectives of $\mathrm{MŁ}_{n}^{c}$ are a unary connective $\square$ and the connectives of $\mathrm{E}_{n}^{c}$. Form $\square$ denotes the set of formulas of $\mathrm{MŁ}_{n}^{c}$.

Definition 5.1. Let $(W, R)$ be a Kripke frame (i.e., $R$ is a relation on a set $W)$. Then, $e$ is a Kripke $\mathbf{n}$-valuation on $(W, R)$ iff $e$ is a function from $W \times$ Form $_{\square}$ to $\mathbf{n}$ which satisfies: For each $w \in W$ and $\varphi, \psi \in \mathbf{F o r m}_{\square}$,

- $e(w, \square \varphi)=\bigwedge\left\{e\left(w^{\prime}, \varphi\right) ; w R w^{\prime}\right\} ;$

- $e(w, \varphi @ \psi)=e(w, \varphi) @ e(w, \psi)$ for $@=\wedge, \vee, *, \wp, \rightarrow$;

- $e\left(w, \varphi^{\perp}\right)=(e(w, \varphi))^{\perp}$;

- $e(w, r)=r$ for $r \in \mathbf{n}$.

Then, $(W, R, e)$ is called an $\mathbf{n}$-valued Kripke model. Define $\mathrm{ME}_{n}^{c}$ as the set of all those formulas $\varphi \in \mathbf{F o r m}_{\square}$ such that $e(w, \varphi)=1$ for any $\mathbf{n}$-valued Kripke model $(W, R, e)$ and any $w \in W$.

By straightforward computation, we have the following lemma. Recall the definition of $\mathrm{U}_{r}$ (Definition 2.8).

Lemma 5.2. Let $\varphi, \psi \in$ Form $_{\square}$ and $r \in \mathbf{n}$. (i) $\mathrm{U}_{r}(\square \varphi) \leftrightarrow \square \mathrm{U}_{r}(\varphi) \in \mathrm{MŁ}_{n}^{c}$. (ii) $\square(\varphi \wedge \psi) \leftrightarrow \square \varphi \wedge \square \psi \in \mathrm{ME}_{n}^{c}$ and $\square 1 \leftrightarrow 1 \in \mathrm{ME}_{n}^{c}$. (iii) $\square(\varphi * \varphi) \leftrightarrow$ $(\square \varphi) *(\square \varphi) \in \mathrm{MŁ}_{n}^{c}$ and $\square(\varphi \wp \varphi) \leftrightarrow(\square \varphi) \wp(\square \varphi) \in \mathrm{MŁ}_{n}^{c}$.

Definition 5.3. For $X \subset$ Form $_{\square}, X$ is satisfiable iff there are an $\mathbf{n}$-valued Kripke model $(W, R, e)$ and $w \in W$ such that $e(w, \varphi)=1$ for any $\varphi \in X$.

$\mathrm{ME}_{n}^{c}$-algebras and homomorphisms are defined as follows.

Definition 5.4. Let $A$ be an $\mathrm{E}_{n}^{c}$-algebra. Then, $(A, \square)$ is an $\mathrm{MŁ}_{n}^{c}$-algebra iff it satisfies the following set of equations: $\left\{\varphi=\psi ; \varphi \leftrightarrow \psi \in \mathrm{ME}_{n}^{c}\right\}$.

A homomorphism of $\mathrm{ME}_{n}^{c}$-algebras is defined as a homomorphism of $\mathrm{E}_{n}^{c}$-algebras which additionally preserves the operation $\square$.

Throughout this paper, we do not distinguish between formulas of $\mathrm{ME}_{n}^{c}$ and terms of $\mathrm{ME}_{n}^{c}$-algebras.

Definition 5.5. Let $A$ be an $\mathrm{MŁ}_{n}^{c}$-algebra. Define a relation $R_{\square}$ on $\operatorname{Spec}(A)$ by

$$
v R_{\square} u \Leftrightarrow \forall r \in \mathbf{n} \forall x \in A(v(\square x) \geq r \text { implies } u(x) \geq r) .
$$

Define $e: \operatorname{Spec}(A) \times A \rightarrow \mathbf{n}$ by $e(v, x)=v(x)$ for $v \in \operatorname{Spec}(A)$ and $x \in A$. Then, $\left(\operatorname{Spec}(A), R_{\square}, e\right)$ is called the $\mathbf{n}$-valued canonical model of $A$. 
Proposition 5.6. Let $A$ be an $\mathrm{ME}_{n}^{c}$-algebra. Then, the $\mathbf{n}$-valued canonical model $\left(\operatorname{Spec}(A), R_{\square}, e\right)$ of $A$ is an $\mathbf{n}$-valued Kripke model. In particular, $e(v, \square x)=v(\square x)=\bigwedge\left\{u(x) ; v R_{\square} u\right\}$ for $x \in A$ and $v \in \operatorname{Spec}(A)$.

Proof. It suffices to show that $e$ is a Kripke $\mathbf{n}$-valuation. Since $v$ is a homomorphism of $\mathrm{E}_{n}^{c}$-algebras, it remains to show $e(v, \square x)=\bigwedge\left\{u(x) ; v R_{\square} u\right\}$. To prove this, it is enough to show that, for any $r \in \mathbf{n}$, (i) $v(\square x) \geq r$ iff (ii) $v R_{\square} u$ implies $u(x) \geq r$. By the definition of $R_{\square}$, (i) implies (ii). We show the converse. To prove the contrapositive, assume $v(\square x) \nsupseteq r$, i.e., $\mathrm{U}_{r}(\square x) \notin v^{-1}(\{1\})$. Let

$$
F_{0}=\left\{\mathrm{U}_{s}(x) ; s \in \mathbf{n} \text { and } \mathrm{U}_{s}(\square x) \in v^{-1}(\{1\})\right\} .
$$

Let $F$ be the $\mathbf{n}$-filter of $A$ generated by $F_{0}$. We claim that $\mathrm{U}_{r}(x) \notin F$. Suppose for contradiction that $\mathrm{U}_{r}(x) \in F$. Then, there is $\varphi \in A$ such that $\varphi \leq \mathrm{U}_{r}(x)$ and $\varphi$ is constructed from $*$ and elements of $F_{0}$. Since $\mathrm{U}_{s}(x)$ is idempotent, $\mathrm{U}_{s_{1}}\left(x_{1}\right) * \mathrm{U}_{s_{2}}\left(x_{2}\right)=\mathrm{U}_{s_{1}}\left(x_{1}\right) \wedge \mathrm{U}_{s_{2}}\left(x_{2}\right)$ and so we may assume that $\varphi=\bigwedge\left\{\mathrm{U}_{s}(x) ; \mathrm{U}_{s}(x) \in F_{1}\right\}$ for some finite subset $F_{1}$ of $F_{0}$. By Lemma 5.2, $\square \varphi=\bigwedge\left\{\mathrm{U}_{s}(\square x) ; \mathrm{U}_{s}(x) \in F_{1}\right\}$. By the definition of $F_{0}$, $\mathrm{U}_{s}(\square x) \in v^{-1}(\{1\})$ for any $\mathrm{U}_{s}(x) \in F_{1}$ and so $\square \varphi \in v^{-1}(\{1\})$. Since $\varphi \leq \mathrm{U}_{r}(x)$, we have $\square \varphi \leq \square \mathrm{U}_{r}(x)=\mathrm{U}_{r}(\square x)$. Thus, $\mathrm{U}_{r}(\square x) \in v^{-1}(\{1\})$, which contradicts $\mathrm{U}_{r}(\square x) \notin v^{-1}(\{1\})$. Hence $\mathrm{U}_{r}(x) \notin F$. By Proposition 2.14, there is a prime $\mathbf{n}$-filter $P$ of $A$ such that $\mathrm{U}_{r}(x) \notin P$ and $F \subset P$. By Proposition 2.17, $v_{P} \in \operatorname{Spec}(A)$. Since $\mathrm{U}_{r}(x) \notin P$, we have $v_{P}(x) \nsupseteq r$. Since $F_{0} \subset F \subset P$, we have $v R_{\square} v_{P}$. Thus, (ii) does not hold.

The following is a compactness theorem for $\mathrm{MŁ}_{n}^{c}$.

Theorem 5.7. Let $X \subset$ Form $_{\square}$. Assume that any finite subset of $X$ is satisfiable. Then, $X$ is satisfiable.

Proof. Let $A$ be the Lindenbaum algebra of $\mathrm{MŁ}_{n}^{c}$. We may consider $X \subset A$. We show that $X$ has f.i.p. with respect to $*$. If not, then there are $n \in \omega$ with $n \neq 0$ and $x_{1}, \ldots, x_{n} \in X$ such that $x_{1} * \ldots * x_{n}=0$, which is a contradiction, since $\left\{x_{1}, \ldots, x_{n}\right\}$ is satisfiable by assumption. Thus, by Proposition 2.16, there is a prime $\mathbf{n}$-filter $P$ of $A$ with $X \subset P$. By Proposition 2.17, $v_{P}$ is a homomorphism, i.e., $v_{P} \in \operatorname{Spec}(A)$. Consider the $\mathbf{n}$-valued canonical model $\left(\operatorname{Spec}(A), R_{\square}, e\right)$ of $A$. Then, $e\left(v_{P}, x\right)=v_{P}(x)=1$ for any $x \in X$ by Proposition 2.17. Thus, $X$ is satisfiable.

Proposition 5.8. Let $A$ be an $\mathrm{ME}_{n}^{c}$-algebra. Then, $\mathcal{B}(A)$ forms a modal algebra. 
Proof. If $x \in A$ is idempotent, then $\square x$ is also idempotent, since $\square x * \square x=$ $\square(x * x)=\square x$ by Lemma 5.2. Thus, $\mathcal{B}(A)$ is closed under $\square$. By Lemma $5.2, \mathcal{B}(A)$ forms a modal algebra.

Definition 5.9. Let $A$ be an $\mathrm{MŁ}_{n}^{c}$-algebra. Define a relation $R_{\square_{2}}$ on $\operatorname{Spec}_{\mathbf{2}}(\mathcal{B}(A))$ by $v R_{\square_{\mathbf{2}}} u \Leftrightarrow \forall x \in \mathcal{B}(A)(v(\square x)=1$ implies $u(x)=1)$.

Proposition 5.10. Let $A$ be an $\mathrm{M七}_{n}^{c}$-algebra. For $v, u \in \operatorname{Spec}(A), v R_{\square} u$ iff $t_{1}(v) R_{\square_{2}} t_{1}(u)$ (for the definition of $t_{1}$, see Proposition 4.20).

Proof. By $\square \mathrm{T}_{1}(x)=\mathrm{T}_{1}(\square x)$, if $v R_{\square} u$ then $t_{1}(v) R_{\square_{2}} t_{1}(u)$. We show the converse. Assume $t_{1}(v) R_{\square_{2}} t_{1}(u)$. In order to show $v R_{\square} u$, it suffices to prove that, for any $r \in \mathbf{n}$ and any $x \in A, v\left(\square \mathrm{U}_{r}(x)\right)=1$ implies $u\left(\mathrm{U}_{r}(x)\right)=$ 1 , which follows from the assumption, since we have $\mathrm{U}_{r}(x) \in \mathcal{B}(A)$ and $\mathrm{T}_{1}\left(\mathrm{U}_{r}(x)\right)=\mathrm{U}_{r}(x)$.

\section{A fuzzy topological duality for $\mathrm{ME}_{n}^{c}$-algebras}

In this section, based on the fuzzy topological duality for $\mathrm{E}_{n}^{c}$-algebras, we show a fuzzy topological duality for $\mathrm{MŁ}_{n}^{c}$-algebras, which is a generalization of Jónsson-Tarski duality for modal algebras via fuzzy topology, where note that $\mathrm{MŁ}_{2}^{c}$-algebras coincide with modal algebras.

Definition 6.1. $\mathrm{MŁ}_{n}^{c}$-Alg denotes the category of $\mathrm{MŁ}_{n}^{c}$-algebras and homomorphisms of $\mathrm{ME}_{n}^{c}$-algebras.

Our aim in this section is to show that the category $\mathrm{ME}_{n}^{c}$-Alg is dually equivalent to the category $\mathrm{FRS}_{n}$, which is defined in Definition 6.3 below.

For a Kripke frame $(S, R)$, we can define a modal operator $\square$ on the "n-valued powerset algebra" $\mathbf{n}^{S}$ of $S$ as follows.

Definition 6.2. Let $(S, R)$ be a Kripke frame and $f$ a function from $S$ to n. Define $\square_{R} f: S \rightarrow \mathbf{n}$ by $\left(\square_{R} f\right)(x)=\bigwedge\{f(y) ; x R y\}$.

Recall: For a Kripke frame $(S, R)$ and an $\mathbf{n}$-fuzzy set $\mu$ on $S$, an $\mathbf{n}$-fuzzy set $R^{-1}[\mu]$ on $S$ is defined by $R^{-1}[\mu](x)=\bigvee\{\mu(y) ; x R y\}$ for $x \in S$.

Definition 6.3. We define the category $\mathrm{FRS}_{n}$ as follows.

An object in $\mathrm{FRS}_{n}$ is a tuple $(S, R)$ such that $S$ is an object in $\mathrm{FBS}_{n}$ and that a relation $R$ on $S$ satisfies the following conditions:

1. if $\forall f \in \operatorname{Cont}(S)\left(\left(\square_{R} f\right)(x)=1 \Rightarrow f(y)=1\right)$ then $x R y$; 
2. if $\mu \in \operatorname{Cont}(S)$, then $R^{-1}[\mu] \in \operatorname{Cont}(S)$.

An arrow $f:\left(S_{1}, R_{1}\right) \rightarrow\left(S_{2}, R_{2}\right)$ in $\mathrm{FRS}_{n}$ is an arrow $f: S_{1} \rightarrow S_{2}$ in $\mathrm{FBS}_{n}$ which satisfies the following conditions:

1. if $x R_{1} y$ then $f(x) R_{2} f(y)$;

2. if $f\left(x_{1}\right) R_{2} x_{2}$ then there is $y_{1} \in S_{1}$ such that $x_{1} R_{1} y_{1}$ and $f\left(y_{1}\right)=x_{2}$.

An object in $\mathrm{FRS}_{n}$ is called an $\mathbf{n}$-fuzzy relational space.

The item 1 in the object part of Definition 6.3 is an $\mathbf{n}$-fuzzy version of the tightness condition of descriptive general frames in classical modal logic (for the definition of the tightness condition in classical modal logic, see [3]).

Definition 6.4. We define a contravariant functor $\mathrm{RSpec}: \mathrm{ME}_{n}^{c}-\mathrm{Alg} \rightarrow$ $\mathrm{FRS}_{n}$. For an object $A$ in $\mathrm{ME}_{n}^{c}$-Alg, define $\operatorname{RSpec}(A)=\left(\operatorname{Spec}(A), R_{\square}\right)$. For an arrow $f: A \rightarrow B$ in $\mathrm{ME}_{n}^{c}$-Alg, define $\operatorname{RSpec}(f): \operatorname{RSpec}(B) \rightarrow \operatorname{RSpec}(A)$ by $\operatorname{RSpec}(f)(v)=v \circ f$ for $v \in \operatorname{Spec}(B)$.

We call $\operatorname{RSpec}(A)$ the relational spectrum of $A$. The well-definedness of RSpec is shown by Proposition 6.6 and Proposition 6.7 below.

Definition 6.5. Let $A$ be an $\mathrm{MŁ}_{n}^{c}$-algebra. Then, we define $\operatorname{RSpec}_{2}(\mathcal{B}(A))$ as $\left(\operatorname{Spec}_{\mathbf{2}}(\mathcal{B}(A)), R_{\square_{2}}\right)$. Let $A_{1}$ and $A_{2}$ be $\mathrm{ME}_{n}^{c}$-algebras and $f: \mathcal{B}\left(A_{1}\right) \rightarrow$ $\mathcal{B}\left(A_{2}\right)$. Then, we define $\operatorname{RSpec}_{2}(f): \operatorname{RSpec}_{2}\left(\mathcal{B}\left(A_{2}\right)\right) \rightarrow \operatorname{RSpec}_{\mathbf{2}}\left(\mathcal{B}\left(A_{1}\right)\right)$ by $\operatorname{RSpec}_{\mathbf{2}}(f)(v)=v \circ f$ for $v \in \operatorname{RSpec}_{\mathbf{2}}\left(\mathcal{B}\left(A_{2}\right)\right)$.

Proposition 6.6. For an $\mathrm{ME}_{n}^{c}$-algebra $A$, $\operatorname{RSpec}(A)$ is an object in $\mathrm{FRS}_{n}$.

Proof. It suffices to show the items 1 and 2 in the object part of Definition 6.3. We first show the item 1 by proving the contrapositive. Assume $(v, u) \notin$ $R_{\square}$, i.e., there are $r \in \mathbf{n}$ and $x \in A$ such that $v(\square x) \geq r$ and $u(x) \nsupseteq r$. By Lemma 2.8, $v\left(\mathrm{U}_{r}(\square x)\right)=1$ and $u\left(\mathrm{U}_{r}(x)\right)=0$. Then, $\left\langle\mathrm{U}_{r}(x)\right\rangle(u)=0$. By Proposition 5.6 and Lemma 5.2,

$$
\left(\square_{R}\left\langle\mathrm{U}_{r}(x)\right\rangle\right)(v)=\bigwedge\left\{\left\langle\mathrm{U}_{r}(x)\right\rangle\left(v^{\prime}\right) ; v R_{\square} v^{\prime}\right\}=v\left(\square \mathrm{U}_{r}(x)\right)=v\left(\mathrm{U}_{r} \square x\right)=1 .
$$

As is shown in the proof of Lemma 4.14, $\left\langle\mathrm{U}_{r}(x)\right\rangle$ is continuous.

We show the item 2. Since Cont $\circ \operatorname{Spec}(A)=\{\langle x\rangle ; x \in A\}$ as is shown in the proof of Lemma 4.14, it suffices to show that, for any $x \in A$, $R_{\square}^{-1}(\langle x\rangle) \in$ ContoSpec $(A)$. Let $\diamond x$ denote $\left(\square\left(x^{\perp}\right)\right)^{\perp}$. Since $\left(R_{\square}^{-1}(\langle x\rangle)\right)(v)=$ $\bigvee\left\{u(x) ; v R_{\square} u\right\}=v(\diamond x)$, we have $R_{\square}^{-1}(\langle x\rangle)=\langle\diamond x\rangle \in \operatorname{Cont} \circ \operatorname{Spec}(A)$. 
Proposition 6.7. For $\mathrm{ME}_{n}^{c}$-algebras $A_{1}$ and $A_{2}$, let $f: A_{1} \rightarrow A_{2}$ be a homomorphism of $\mathrm{ME}_{n}^{c}$-algebras. Then, $\operatorname{RSpec}(f)$ is an arrow in $\mathrm{FRS}_{n}$.

Proof. Define $f_{*}: \mathcal{B}\left(A_{1}\right) \rightarrow \mathcal{B}\left(A_{2}\right)$ by $f_{*}(x)=f(x)$ for $x \in \mathcal{B}\left(A_{1}\right)$. By Proposition 5.8, $f_{*}$ is a homomorphism of modal algebras. Consider $\operatorname{RSpec}_{\mathbf{2}}\left(f_{*}\right)$ : $\operatorname{RSpec}_{\mathbf{2}}\left(\mathcal{B}\left(A_{2}\right)\right) \rightarrow \operatorname{RSpec}_{\mathbf{2}}\left(\mathcal{B}\left(A_{1}\right)\right)$. By Jónsson-Tarski duality for modal algebras (see $[16,1]), \operatorname{RSpec}_{2}\left(f_{*}\right)$ is an arrow in $\mathrm{FRS}_{2}$.

We first show that $\operatorname{RSpec}(f)$ satisfies the item 2 in the arrow part of Definition 6.3. Assume $\operatorname{RSpec}(f)\left(v_{2}\right) R_{\square} u_{1}$ for $v_{2} \in \operatorname{RSpec}\left(A_{2}\right)$ and $u_{1} \in$ $\operatorname{RSpec}\left(A_{1}\right)$. By Proposition 5.10, $t_{1}\left(\operatorname{RSpec}(f)\left(v_{2}\right)\right) R_{\square_{2}} t_{1}\left(u_{1}\right)$. It follows from $t_{1}\left(\operatorname{RSpec}(f)\left(v_{2}\right)\right)=\mathrm{T}_{1} \circ v_{2} \circ f=\operatorname{RSpec}_{2}\left(f_{*}\right)\left(t_{1}\left(v_{2}\right)\right)$ that we have $\operatorname{RSpec}_{2}\left(f_{*}\right)\left(t_{1}\left(v_{2}\right)\right) R_{\square_{2}} t_{1}\left(u_{1}\right)$. Since $\operatorname{RSpec}_{2}\left(f_{*}\right)$ is an arrow in $\mathrm{FRS}_{2}$, there is $u_{2} \in \operatorname{RSpec}_{\mathbf{2}}\left(\mathcal{B}\left(A_{2}\right)\right)$ such that $t_{1}\left(v_{2}\right) R_{\square_{2}} u_{2}$ and $\operatorname{RSpec}_{2}\left(f_{*}\right)\left(u_{2}\right)=t_{1}\left(u_{1}\right)$. Define $u_{2}^{\prime} \in \operatorname{RSpec}\left(A_{2}\right)$ by $u_{2}^{\prime}(x)=r \Leftrightarrow u_{2}\left(\mathrm{~T}_{r}(x)\right)=1$. It is verified in a similar way to Proposition 2.17 that $u_{2}^{\prime}$ is a homomorphism.

We claim that $v_{2} R_{\square} u_{2}^{\prime}$ and $\operatorname{RSpec}(f)\left(u_{2}^{\prime}\right)=u_{1}$. Let $x \in A_{2}$ and $r \in \mathbf{n}$. If $v_{2}(\square x) \geq r$ then $\left(t_{1}\left(v_{2}\right)\right)\left(\square \mathrm{U}_{r}(x)\right)=1$ and, since $t_{1}\left(v_{2}\right) R_{\square_{2}} u_{2}$, we have $u_{2}\left(\mathrm{U}_{r}(x)\right)=1$, whence $u_{2}^{\prime}(x) \geq r$. Thus, $v_{2} R_{\square} u_{2}^{\prime}$. Next we show $\operatorname{RSpec}(f)\left(u_{2}^{\prime}\right)=u_{1}$. Let $r=\left(\operatorname{RSpec}(f)\left(u_{2}^{\prime}\right)\right)(x)$ for $x \in A_{1}$. Then, $u_{2}\left(\mathrm{~T}_{r}(f(x))\right)=1$ and so $\left(\operatorname{RSpec}_{\mathbf{2}}\left(f_{*}\right)\left(u_{2}\right)\right)\left(\mathrm{T}_{r}(x)\right)=1$. It follows from $\operatorname{RSpec}_{2}\left(f_{*}\right)\left(u_{2}\right)=t_{1}\left(u_{1}\right)$ that $\left(t_{1}\left(u_{1}\right)\right)\left(\mathrm{T}_{r}(x)\right)=1$ and so $u_{1}\left(\mathrm{~T}_{r}(x)\right)=1$, whence $u_{1}(x)=r=\left(\operatorname{RSpec}(f)\left(u_{2}^{\prime}\right)\right)(x)$. Thus $\operatorname{RSpec}(f)$ satisfies the item 2 .

It is easier to verify that $\operatorname{RSpec}(f)$ satisfies the item 1 in the arrow part of Definition 6.3.

Definition 6.8. A contravariant functor MCont : $\mathrm{FRS}_{n} \rightarrow \mathrm{ME}_{n}^{c}$-Alg is defined as follows. For an object $(S, R)$ in $\operatorname{FRS}_{n}$, define $\operatorname{MCont}(S, R)=$ $\left(\operatorname{Cont}(S), \square_{R}\right)$. For an arrow $f:\left(S_{1}, R_{1}\right) \rightarrow\left(S_{2}, R_{2}\right)$ in $\mathrm{FRS}_{n}$, define $\operatorname{MCont}(f): \operatorname{MCont}\left(S_{2}, R_{2}\right) \rightarrow \operatorname{MCont}\left(S_{1}, R_{1}\right)$ by $\operatorname{MCont}(f)(g)=g \circ f$ for $g \in \operatorname{Cont}\left(S_{2}\right)$.

The well-definedness of MCont is shown by the following propositions.

Proposition 6.9. For an object $(S, R)$ in $\operatorname{FRS}_{n}, \operatorname{MCont}(S, R)$ is an $\mathrm{MŁ}_{n}^{c}-$ algebra.

Proof. We first show that if $f \in \operatorname{Cont}(S)$ then $\square_{R} f \in \operatorname{Cont}(S)$. Let $f \in$ $\operatorname{Cont}(S)$ and $\mu$ an open $\mathbf{n}$-fuzzy set on $\mathbf{n}$. Define $\mu_{r}$ as in the proof of Lemma 4.3 and then it suffices to show that $\left(\square_{R} f\right)^{-1}\left(\mu_{r}\right)$ is an open $\mathbf{n}$-fuzzy set on $S$ for any $r \in \mathbf{n}$. By Lemma 2.8,

$$
\left(\square_{R} f\right)^{-1}\left(\mu_{r}\right)=R^{-1}\left[\mu_{r} \circ f\right] \wedge\left(R^{-1}\left[\left(\mathrm{U}_{r} \circ f\right)^{\perp}\right]\right)^{\perp} .
$$


Since both $\mu_{r} \circ f$ and $\left(\mathrm{U}_{r} \circ f\right)^{\perp}$ are elements of $\operatorname{Cont}(S)$, the right-hand side is an element of $\operatorname{Cont}(S)$ by the definition of $R$ and so is an open $\mathbf{n}$-fuzzy set on $S$, since $S$ is zero-dimensional. Thus $\square_{R} f \in \operatorname{Cont}(S)$.

Next we show that $\operatorname{MCont}(S, R)$ satisfies $\left\{\varphi=\psi ; \varphi \leftrightarrow \psi \in \operatorname{MŁ}_{n}^{c}\right\}$. Consider $\operatorname{Cont}(S)$ as the set of propositional variables. Since $\operatorname{Cont}(S)$ is closed under the operations of $\operatorname{Cont}(S)$, an element of Form $_{\square}$ may be seen as an element of $\operatorname{Cont}(S)$. Define $e: S \times \mathbf{F o r m}_{\square} \rightarrow \mathbf{n}$ by $e(w, f)=f(w)$ for $w \in S$ and $f \in \operatorname{Cont}(S)$. Then, $(S, R, e)$ is an $\mathbf{n}$-valued Kripke model by the definition of the operations of $\operatorname{Cont}(S)$. Since $e(w, f)=1$ for any $w \in S$ iff $f=1$, it follows from the definition of $\operatorname{MŁ}_{n}^{c}$ that $\operatorname{MCont}(S, R)$ satisfies $\left\{\varphi=\psi ; \varphi \leftrightarrow \psi \in \mathrm{ME}_{n}^{c}\right\}$.

Proposition 6.10. Let $f:\left(S_{1}, R_{1}\right) \rightarrow\left(S_{2}, R_{2}\right)$ be an arrow in $\mathrm{FRS}_{n}$. Then, $\mathrm{MCont}(f)$ is a homomorphism of $\mathrm{ME}_{n}^{c}$-algebras.

Proof. It remains to show that $\operatorname{MCont}(f)\left(\square g_{2}\right)=\square\left(\operatorname{MCont}(f)\left(g_{2}\right)\right)$ for $g_{2} \in$ $\operatorname{Cont}\left(S_{2}\right)$. For $x_{1} \in S_{1},\left(\operatorname{MCont}(f)\left(\square g_{2}\right)\right)\left(x_{1}\right)=\bigwedge\left\{g_{2}\left(y_{2}\right) ; f\left(x_{1}\right) R_{2} y_{2}\right\}$. Let $a$ denote the right-hand side. We also have $\left(\square\left(\operatorname{MCont}(f)\left(g_{2}\right)\right)\right)\left(x_{1}\right)=$ $\bigwedge\left\{g_{2}\left(f\left(y_{1}\right)\right) ; x_{1} R_{1} y_{1}\right\}$. Let $b$ denote the right-hand side. Since $x_{1} R_{1} y_{1}$ implies $f\left(x_{1}\right) R_{1} f\left(y_{1}\right)$, we have $a \leq b$. By the item 2 in the arrow part of Definition 6.3, we have $a \geq b$. Hence $a=b$.

Theorem 6.11. Let $A$ be an object in $\mathrm{ME}_{n}^{c}$-Alg. Then, $A$ is isomorphic to $\mathrm{MCont} \circ \mathrm{RSpec}(A)$ in the category $\mathrm{MŁ}_{n}^{c}$-Alg.

Proof. We claim that $\langle-\rangle: A \rightarrow \operatorname{MCont} \circ \operatorname{RSpec}(A)$ is an isomorphism of $\mathrm{ME}_{n}^{c}$-algebras. By Theorem 4.21, it remains to show that $\langle\square x\rangle=\square_{R_{\square}}\langle x\rangle$ for $x \in A$. By Proposition 5.6, we have the following for $v \in \operatorname{Spec}(A)$ : $\left(\square_{R_{\square}}\langle x\rangle\right)(v)=\bigwedge\left\{u(x) ; v R_{\square} u\right\}=v(\square x)=\langle\square x\rangle(v)$.

Theorem 6.12. Let $(S, R)$ be an object in $\mathrm{FRS}_{n}$. Then, $(S, R)$ is isomorphic to RSpec o $\operatorname{MCont}(S, R)$ in the category $\operatorname{FRS}_{n}$.

Proof. Define $\Phi:(S, R) \rightarrow \operatorname{RSpec} \circ \operatorname{MCont}(S, R)$ by $\Phi(x)(f)=f(x)$ for $x \in S$ and $f \in \operatorname{Cont}(S)$. We show: For any $x, y \in S, x R y$ iff $\Phi(x) R_{\square_{R}} \Phi(y)$. Assume $x R y$. Let $r \in \mathbf{n}$ and $f \in \operatorname{Cont}(S)$ with $\Phi(x)\left(\square_{R} f\right) \geq r$. Since $\Phi(x)\left(\square_{R} f\right)=\bigwedge\{f(z) ; x R z\}$, we have $\Phi(y)(f)=f(y) \geq r$. Next we show the converse. To prove the contrapositive, assume $(x, y) \notin R$. By Definition 6.3, there is $f \in \operatorname{Cont}(S)$ such that $\left(\square_{R} f\right)(x)=1$ and $f(y) \neq 1$. Then, $\Phi(x)\left(\square_{R} f\right)=1$ and $\Phi(y)(f) \neq 1$. Thus, we have $(\Phi(x), \Phi(y)) \notin R_{\square_{R}}$.

By Theorem 4.22, it remains to prove that $\Phi$ and $\Phi^{-1}$ satisfy the item 2 in the arrow part of Definition 6.3, which follows from the above fact that $x R y$ iff $\Phi(x) R_{\square_{R}} \Phi(y)$, since $\Phi$ is bijective. 
By Theorem 6.11 and Theorem 6.12 , we obtain a fuzzy topological duality for $\mathrm{MŁ}_{n}^{c}$-algebras, which is a generalization of Jónsson-Tarski duality for modal algebras to the $n$-valued case via fuzzy topology.

Theorem 6.13. The category $\mathrm{M}_{n}^{c}$-Alg is dually equivalent to the category $\mathrm{FRS}_{n}$ via the functors $\mathrm{RSpec}(-)$ and $\mathrm{MCont}(-)$.

Proof. By arguing as in the proof of Theorem 4.23, this theorem follows immediately from Theorem 6.11 and Theorem 6.12 .

Acknowledgements. The author would like to thank an anonymous referee for helpful comments.

\section{References}

[1] P. Blackburn, M. de Rijke and Y. Venema, Modal logic, CUP, 2001.

[2] V. Boicescu, A. Filipoiu, G. Georgescu and S. Rudeanu, Lukasiewicz-Moisil algebras, North-Holland Publishing Co., 1991.

[3] A. Chagrov and M. Zakharyaschev, Modal logic, OUP, 1997.

[4] C. C. Chang, Algebraic analysis of many-valued logics, Trans. Amer. Math. Soc. 88 (1958) 476-490.

[5] C. L. Chang, Fuzzy topological spaces, J. Math. Anal. Appl. 24 (1968) 182-190.

[6] R. L. O. Cignoli, I. M. L. D'Ottaviano and D. Mundici, Algebraic foundations of many-valued reasoning, Kluwer Academic Publishers, 1999.

[7] R. L. O. Cignoli, E. J. Dubuc, and D. Mundici, Extending Stone duality to multisets and locally finite MV-algebras. J. Pure Appl. Algebra, 189 (2004) $37-59$.

[8] A. Di Nola and P. Niederkorn, Natural dualities for varieties of BL-algebras, Arch. Math. Log. 44 (2005) 995-1007.

[9] M. C. Fitting, Many-valued modal logics, Fund. Inform. 15 (1991) 235-254.

[10] M. C. Fitting, Many-valued modal logics II, Fund. Inform. 17 (1992) 55-73.

[11] J. A. Goguen, L-fuzzy sets, J. Math. Anal. Appl. 18 (1967) 145-174.

[12] J. A. Goguen, The fuzzy Tychonoff theorem, J. Math. Anal. Appl. 43 (1973) 734-742.

[13] S. Gottwald, A treatise on many-valued logics, Research Studies Press, 2001.

[14] R. Grigolia, Algebraic analysis of Łukasiewicz-Tarski n-valued logical systems, Selected papers on Eukasiewicz sentential calculi, Wroclaw, pp. 81-91, 1977. 
[15] P. Hájek, Metamathematics of fuzzy logic, Kluwer Academic Publishers, 1998.

[16] G. Hansoul, A duality for Boolean algebras with operators, Algebra Universalis 17 (1983) 34-49.

[17] P. T. Johnstone; Stone spaces, CUP, 1986.

[18] L. Leustean, Sheaf representations of BL-algebras, Soft Computing 9 (2005) 897-909.

[19] Y. M. Liu and M. K. Luo, Fuzzy topology, World Scientific, 1998.

[20] J. Łukasiewicz and A. Tarski, Untersuchungen über den Assagenkalkul, Compt. Rend. des Séances Société des Sciences et Lettres de Varsovie Classe III 23 (1930) 3-50.

[21] G. Malinowski, Many-valued logics, Clarendon Press, 1993.

[22] Y. Maruyama, Algebraic study of lattice-valued logic and lattice-valued modal logic, Lecture Notes in Computer Science 5378 (2009) 172-186.

[23] Y. Maruyama, A duality for algebras of lattice-valued modal logic, Lecture Notes in Computer Science 5514 (2009) 281-295

[24] P. Niederkorn, Natural dualities for varieties of MV-algebras, J. Math. Anal. Appl. 255 (2001) 58-73.

[25] S. E. Rodabaugh and E. P. Klement (eds.), Topological and algebraic structures in fuzzy sets, Kluwer Academic Publishers, 2003.

[26] G. Sambin and V. Vaccaro, Topology and duality in modal logic, Ann. Pure Appl. Logic 37 (1988) 249-296.

[27] A. P. Sostak, Basic structures of fuzzy topology, Journal of Mathematical Sciences 78 (1996) 662-701.

[28] M. H. Stone, The representation of Boolean algebras, Bull. Amer. Math. Soc. 44 (1938) 807-816

[29] B. Teheux, A duality for the algebras of a Łukasiewicz n+1-valued modal system, Studia Logica 87 (2007) 13-36.

[30] L.A. Zadeh, Fuzzy sets, Information and Control 8 (1965) 338-353. 